\title{
KAC-MOODY GROUPS, INFINITE DIMENSIONAL DIFFERENTIAL GEOMETRY AND CITIES*
}

\author{
WALTER FREYN ${ }^{\dagger}$
}

\begin{abstract}
Minimal affine Kac-Moody groups act on affine twin buildings by isometries. However there is no way to extend this action to any completion of the Kac-Moody groups. To remedy this, we introduce in this paper affine twin cities, a new class of objects, whose elements behave like completions of twin buildings. Twin cities are defined as special arrays of affine buildings connected among themselves by twinnings. Corresponding to completed affine Kac-Moody groups they are characterized by the type of the affine buildings and by some kind of "regularity conditions" describing the completion. The isometry groups of affine twin cities are (completions of) affine Kac-Moody groups. We study applications of cities in infinite dimensional differential geometry by proving infinite dimensional versions of classical differential geometric results: For example, we show that points in an isoparametric submanifold in a Hilbert space correspond to all chambers in a city. In two sequels we will describe the theory of twin cities for formal completions.
\end{abstract}

Key words. Loop group, affine Kac-Moody group, twin city, twin building, polar action, KacMoody geometry.

AMS subject classifications. 17B65, 20G44, 20E42, 22E65, 22E67, 58B99.

1. Introduction. The theme of affine Kac-Moody geometry is the investigation of the geometry lurking behind completions of affine Kac-Moody algebras and Kac-Moody groups. Affine Kac-Moody groups can be described as certain torus extensions $\widehat{L}(G, \sigma)$ of (possibly twisted) loop groups $L(G, \sigma)$. Correspondingly affine Kac-Moody algebras are 2-dimensional extensions of (possibly twisted) loop algebras $L(\mathfrak{g}, \sigma)$. Here $G$ denotes a compact or complex simple Lie group, $\mathfrak{g}$ its Lie algebra and $\sigma \in \operatorname{Aut}(G)$ a diagram automorphism, defining the "twist". Depending on the regularity assumptions on the loops (e.g. of Sobolev class $H^{k}$ ), one gets families of completions of the minimal (=algebraic) affine Kac-Moody groups ${\widehat{L_{a l g} G}}^{\sigma}$. The minimal algebraic loop group just consists of Laurent polynomials. Following Jacques Tits, completions defined by imposing regularity conditions on the loops are called "analytic completions" in contrast to the more abstract formal completion. Various analytic completions and objects closely related to them play an important role in different branches of mathematics and physics, especially quantum field theory, integrable systems and differential geometry. In most cases their use is motivated by the requirement to use functional analytic methods or by the need to work with manifolds and Lie groups [PS86], [Gue97], [SW85], [Tsv03], [Pop05], [PT88], [Kob11], [KW09], [HPTT95], [HL99], [Hei06] and references therein. In this article we introduce affine twin cities as the appropriate generalization of twin buildings in the realm of completions of affine Kac-Moody groups.

To describe the scope of the theory and the content of this article let us start with some general remarks: From an algebraic point of view affine Kac-Moody algebras and simple Lie algebras are closely related as they are both realization of (generalized) Cartan matrices [Kac90], [Car02]. As a consequence, they share important parts of their structure theories. The philosophy of Kac-Moody geometry asserts that this similarity extends to all types of geometric objects whose structure reflects actions

\footnotetext{
*Received August 18, 2011; accepted for publication December 16, 2011.

${ }^{\dagger}$ Mathematisches Institut, Universität Münster, Einsteinstrasse 62, 48149 Münster, Germany (walter.freyn@uni-muenster.de).
} 
of those groups. More precisely Kac-Moody geometry claims the existence of infinite dimensional counterparts to all finite dimensional differential geometry objects, whose symmetries are described by real or complex simple Lie groups [Hei06], [Fre11b]. The symmetries of those infinite dimensional objects are supposed to be the corresponding complex or real affine Kac-Moody groups. Thus, let us start by recalling the finite dimensional blueprint.

In this case the symmetry group is a simple complex or real Lie group. A complex simple Lie group has up to conjugation a unique compact real from. All further real forms are non-compact. Attached to any compact or complex simple Lie group, or more generally to any symmetric space, there is a spherical building [AB08]. Combinatorically a building is described as a simplicial complex or as a graph, satisfying several axioms. Geometrically the building corresponding to a symmetric space $M$ has a clear intuitive description [BH99]: suppose the dimension $k$ of maximal flats (maximal flat subspaces), called the rank, satisfies $k \geq 2$ (otherwise the building degenerates to a set of points) and choose a point $p \in M$. The building can be seen in the tangent space $T_{p} M$ as follows: Consider first all $k$-dimensional tangent spaces to maximal flats through the point $p$ (for a compact simple Lie group choose $p=I d$ the identity, then $T_{p} M$ can be identified with the Lie algebra, maximal flats through the identity are maximal tori and the tangent spaces to maximal flats are just Abelian subalgebras of the Lie algebra $\mathfrak{g}$ ) and remove all intersection points (the so-called singular points). Then each flat is partitioned into chambers and the set of all those chambers for all flats through $p$ corresponds exactly to the chambers of the building. All other cells of the building can be seen in the set of singular points. Chambers belonging to one flat form an apartment. Nevertheless, in this description, one does not see all apartments. To this end one has to pass to the sphere at infinity, to disenthral the construction from the dependence of a base point.

Via the geometric description of the building in the tangent space the connections between the building and polar actions become manifest: Let $M$ be a symmetric space and $p \in M$. The isotropy representation of $M$ is the representation of $K$, the group of all isometries of $M$ fixing $p$ on $T_{p} M$ :

$$
K: T_{p} M \longrightarrow T_{p} M,
$$

For compact Lie groups the isotropy representation coincides with the Adjoint representation.

The principal orbits of the isotropy representation have the following properties:

1. The principal orbits meet the tangent spaces to the flats orthogonally and have complementary dimension. In particular, the normal spaces to the orbits are integrable. Actions with this property are called polar. Conversely, any polar action is orbit equivalent to the isotropy representation of a symmetric space [BCO03].

2. The geometry of the principal orbits as submanifolds of Euclidean space is particularly nice and simple. They are so-called isoparametric submanifolds [PT88]. Conversely, by a theorem of Thorbergsson [Tho91], isoparametric submanifolds are principal orbits of polar representations if they are full, irreducible and the codimension is not 2 .

To summarize the finite dimensional blueprint:

- Polar representations correspond to symmetric spaces, isoparametric submanifolds correspond roughly to polar representations. 
- Chambers in buildings correspond to points in isoparametric submanifolds.

- Buildings describe the structure at infinity of symmetric spaces of noncompact type.

- The geometric realization of buildings can be equivariantly embedded via the isotropy representation into the tangent space of a symmetric space.

Let us now turn to the infinite dimensional counterpart: Affine twin buildings have been investigated by Ronan and Tits as a tool to understand minimal affine Kac-Moody groups [Ron03]. They play a similar role for affine Kac-Moody groups as classical buildings play for finite dimensional semisimple Lie groups.

Affine Kac-Moody groups are distinguished among other classes of infinite dimensional Lie groups by two important properties:

- They share the most important structure properties of finite dimensional simple Lie groups, i.e. they have $B N$-pairs, Iwasawa and Bruhat decompositions and highest weight representations [PS86], [Kac90], [Kum02], [MP95]. The main differences can be traced back to the Weyl group being finite for Lie groups and infinite for Kac-Moody groups.

- They have good explicit linear realizations in terms of 2-dimensional extensions of loop algebras and loop groups; thus they allow the use of functional analytic methods and the definition of manifold structures. The resulting Kac-Moody algebras and Kac-Moody groups are called of "analytic" type, in contrast for example to formal completions [Tit84]. Depending on the type of completions, analytic Kac-Moody algebras (groups) are Hilbert-, Banach-, Fréchet-, etc. Lie algebras (groups) [PS86].

Work done during the last 25 years by various authors, notably Ernst Heintze and Chuu-Lian Terng shows that analytic completions of affine Kac-Moody groups describe the symmetries of various interesting objects, that have finite dimensional counterparts, thus confirming the philosophy of Kac-Moody geometry [PS86], [Hei06], [Fre11b], [KW09], [Ter89], [Ter95], [SW85], [Pop06], [HPTT95], [HL99], [Fre09].

For example, it is well known that affine analytic Kac-Moody groups are symmetry groups of (Kac-Moody) symmetric spaces [Fre09]. The structure theory and the classification of those spaces parallels closely the theory of finite dimensional Riemannian symmetric spaces, making them the appropriate generalization. Furthermore there is a theory of polar actions on Hilbert spaces [Ter95] and proper Fredholm isoparametric submanifolds in Hilbert spaces [Ter89]. Principal orbits of polar action on Hilbert spaces are proper Fredholm isoparametric submanifolds in Hilbert spaces. Thus a broad generalization of the finite dimensional blueprint appears. We find the same classes of objects, satisfying similar relations among each other and sharing similar structure properties [Hei06] [Fre11b]. Nevertheless, a serious gap in the infinite dimensional theory described so far is the lack of an object generalizing spherical buildings to Kac-Moody geometry. For algebraic Kac-Moody groups there are algebraic twin buildings, but they do not work well with completions as the action of any completed Kac-Moody group does not preserve the twinning; thus affine twin buildings are of restricted use in the realm of a Kac-Moody geometry. This problem can be seen also on the group level, as analytic Kac-Moody groups do not allow for the definition of a twin $B N$-pair or a $B N$-pair [AB08], [PS86].

The main purpose of this article is to find a solution for this problem. To this end we define a new structure, called twin cities, which generalizes the twin building. Remark that we called cities "universal geometric twin buildings" in [Fre09] and [Fre11b], but changed the denomination, being adverted of the risk of confusion with 
the universal "algebraic" twin building, introduced by Ronan and Tits [RT94]. A twin city consists of two (usually uncountable) families of Euclidean buildings, denoted $\mathfrak{B}^{+}$and $\mathfrak{B}^{-}$, such that each building from one family is twinned with each building from the other family. The family of buildings becomes richer and richer, the weaker the regularity of the loops is assumed. Thus twin cities also reflect the regularity of the corresponding affine Kac-Moody group. For minimal Kac-Moody groups the two families just reduce to one building each. Hence in this special case we recover twin buildings. More precisely, we have the following result:

TheOREm 1.1 (Twin cities). For each analytic Kac-Moody group $\mathcal{G}$ there exists an associated twin city $\mathfrak{B}=\mathfrak{B}^{+} \cup \mathfrak{B}^{-}$, such that

(i) Each connected component $\Delta \in \mathfrak{B}$ is an affine building.

(ii) Each pair $\left(\Delta^{+}, \Delta^{-}\right) \in \mathfrak{B}^{+} \cup \mathfrak{B}^{-}$, consisting of a building $\Delta^{+}$in $\mathfrak{B}^{+}$("positive" building) and a building $\Delta^{-}$in $\mathfrak{B}^{-}$("negative" building), is an affine twin building.

(iii) $\mathcal{G}$ acts on its twin city $\mathfrak{B}$ by isometries.

(iv) "Small" twin cities, associated to Kac-Moody groups, defined by stronger regularity conditions, embed into "big" twin cities, associated to Kac-Moody groups, defined by weaker regularity conditions.

Like the spherical buildings associated to finite dimensional symmetric spaces, one can realize the twin city associated to an affine Kac-Moody symmetric space (group) in its tangent space (Lie algebra). This realization is constructed via the isotropy representation. Thus twin cities occur "in nature". This is the main topic of the second part of the paper. Recall that the natural bilinear form of an affine Kac-Moody algebra is of Lorentz-type. Denote by $c$ and $d$ the generators of the 2-dimensional extension of the loop algebra. $c$ and $d$ have both length 0 (they are "light like"). The loops algebra $\widehat{L}(\mathfrak{g}, \sigma)$ is "space like". Thus the set $\mathfrak{H}_{r}$ of vectors of length $r$ is in fact an infinite dimensional hyperbolic space with two sheets, say $\mathfrak{H}_{r}^{ \pm}$. The tangent spaces to the flats are still finite dimensional and decompose each sheet $\mathfrak{H}_{r}^{ \pm}$into apartments and chambers as in finite dimensions. These are moreover related, since each flat intersects both $\mathfrak{H}_{r}^{+}$and $\mathfrak{H}_{r}^{-}$, which finally yields the twinning. But it turns out that the corresponding "affine buildings" in $\mathfrak{H}_{r}^{+}$and $\mathfrak{H}_{r}^{-}$are not connected, more precisely not any two chambers are connected by a gallery. First of all $\mathfrak{H}_{r}^{ \pm}$both decompose into families $\mathfrak{H}_{\ell, r}^{ \pm}$of parallel horospheres, which can be identified with vector spaces and each connected component of the buildings lies in one horosphere $\mathfrak{H}_{\ell, r}^{ \pm}$. Each horosphere $\mathfrak{H}_{r, \ell}^{ \pm}$decomposes into a family of buildings, whose size depends on the regularity, scilicet into a city.

More precisely we have the following result:

Theorem 1.2 (Embedding of cities). Denote by $\mathfrak{H}_{\ell, r}$ the intersection of the sphere of radius $l \in \mathbb{R}$ of a real affine Kac-Moody algebra $\widehat{L}(\mathfrak{g}, \sigma)$ with the horospheres $r_{d}= \pm r \neq 0$, where $r_{d} \in \mathbb{R}$ is the coefficient of $d$ in $\widehat{L}(\mathfrak{g}, \sigma)$. There is a 2-parameter family $\varphi_{\ell, r},(l, r) \in \mathbb{R} \times \mathbb{R}^{+}$of $\widehat{L}(G, \sigma)$-equivariant immersions of the twin city $\mathfrak{B}^{+} \cup \mathfrak{B}^{-}$into $\widehat{L}(\mathfrak{g}, \sigma)$. $\varphi_{\ell, r}$ embeds the geometric realization of $\mathfrak{B}$ into $\mathfrak{H}_{\ell, r}$. The two parts of the city $\mathfrak{B}^{+}$and $\mathfrak{B}^{-}$are embeds into the two sheets of $H_{\ell, r}$ described by $r_{d}<0$ resp. $r_{d}>0$ of the space $\mathfrak{H}_{\ell, r}$.

We describe the content of this article in more detail:

In section 2 we introduce various common regularity conditions and study the embeddings of smaller Kac-Moody groups (defined using stronger regularity conditions) 
into bigger ones (defined using weaker regularity conditions). We call a subgroup of $L(G, \sigma)$, which is isomorphic to $L_{\mathrm{alg}} G^{\sigma}$, a quasi-algebraic group. The main result of section 2 is that all quasi-algebraic subgroups are conjugate. Furthermore we study the relationship between Borel subgroups in $L_{\mathrm{alg}} G^{\sigma}$ and Borel subgroups in $L(G, \sigma)$ (resp. $\widehat{L}(G, \sigma)$ ).

Section 3 is devoted to a short summary of the theory of affine twin buildings.

Section 4 contains the core of the article: we define geometric $B N$-pairs and their cities, which are the generalizations of $B N$-pairs and buildings to completed groups, and study group actions on them.

In section 5 we describe the connection between polar actions and cities. In particular we prove that points in isoparametric proper Fredholm submanifolds in a Hilbert space, as introduced in [Ter89], correspond to chambers in the twin city of $H^{1}$-regularity. We start with the investigation of the case of Kac-Moody groups and extend the result in a second step to include all $s$-representations of affine Kac-Moody algebras.

In section 6 we investigate the topology of the space of chambers, the space of affine buildings and the twin city. We define an ultrametric pseudo distance on the space of buildings of a city.

In section 7 we describe in detail the twin city of type $A_{1}^{(1)}$.

Two sequels to this paper will be devoted to the description of the spherical building at infinity, develop an abstract theory of cities and investigate the relationship with universal algebraic twin buildings, introduced by Marc Ronan and Jacques Tits in [RT94] and [RT99].

2. Regularity and Kac-Moody theory. To an affine Cartan matrix and a field $\mathbb{F} \in\{\mathbb{R}, \mathbb{C}\}$ there is attached a great variety of different but closely related infinite dimensional Lie algebras: The minimal one is the algebraic Kac-Moody algebra, corresponding to a Lie algebra of polynomial maps. All further Kac-Moody algebras arise as completions: On the one hand, understanding this algebra as an extension of a Lie algebra over the abstract polynomial ring $\mathbb{F}\left[t, t^{-1}\right]$, we can turn to the formal completion and study the resulting Kac-Moody algebras. On the other hand, taking the point of view of polynomial maps on $S^{1}$ resp. $\mathbb{C}^{*}$, we get a great variety of "analytic" completions and Kac-Moody algebras associated to them [Tit84].

Let $\mathfrak{g}$ be a simple complex or compact Lie algebra and $\sigma$ a diagram automorphism. Denote the associated loop algebra by

$$
L(\mathfrak{g}, \sigma):=\{f: \mathbb{R} \longrightarrow \mathfrak{g} \mid f(t+2 \pi)=\sigma f(t), f \text { satisfies some regularity condition }\}
$$

The associated Kac-Moody algebra is defined by $\widehat{L}(\mathfrak{g}, \sigma)=L(\mathfrak{g}, \sigma) \oplus \mathbb{F} c \oplus \mathbb{F} d$ where $d$ acts on $L(\mathfrak{g}, \sigma)$ as a derivative and $c$ is a central element. Hence $[d, f]=f^{\prime}$, $[c, d]=[c, f]=0$ and $[f, g]=[f, g]_{0}+\omega(f, g) c$, where $[f, g]_{0}$ denotes the Lie bracket of $L(\mathfrak{g}, \sigma)$ and $\omega$ is a certain antisymmetric 2 -form. We define the derived algebra as

$$
\widetilde{L}(\mathfrak{g}, \sigma)=[\widehat{L}(\mathfrak{g}, \sigma), \widehat{L}(\mathfrak{g}, \sigma)] \cong L(\mathfrak{g}, \sigma) \oplus \mathbb{R} c .
$$

Similarly we can attach a variety of different Kac-Moody groups to a given affine root datum. In his overview [Tit84], Jacques Tits describes realizations of a root datum at the algebraic, the formal and the analytic level. In this article, aside from the algebraic Kac-Moody groups, we will encounter various Kac-Moody groups of the 
analytic level. These groups are closely related: The algebraic one is contained in all other Kac-Moody groups. At the analytic level, Kac-Moody groups defined using stronger regularity conditions embed naturally into Kac-Moody groups defined using weaker regularity conditions. Nevertheless, all those embeddings are by no means unique.

Similarly to affine Kac-Moody algebras that can be viewed as 2-dimensional extensions of certain loop algebras, affine Kac-Moody groups can be realized as torus extensions $\widehat{L}(G, \sigma)$ of groups of maps

$$
L(G, \sigma)=\{f: \mathbb{R} \longrightarrow G \mid f(t+2 \pi)=\sigma f(t), f \text { satisfies some regularity condition }\},
$$

where $G$ denotes a simple complex or compact Lie group and $\sigma$ a diagram automorphism of $G$. We construct first $\widetilde{L}(G, \sigma)$ as a central $S^{1}$ - resp. $\mathbb{C}^{*}$-extension, corresponding to the $c$-term of the Kac-Moody algebra, then we take a semidirect product with $S^{1}$ resp. $\mathbb{C}^{*}$ whose action on $L(G, \sigma)$ is defined to be a shift of the argument: $w \cdot f(t)=f(t+w)$. For this shift to be well defined we need in the complex setting a continuation of $f$ to $\mathbb{C}^{*}$ For details see [PS86], [Pop05], [Fre09], [KW09] and various other references there-in.

Depending on their loop realizations, affine Kac-Moody algebras (resp. groups) break up into two subclasses: the twisted ones, i.e. those with $\sigma \neq \mathrm{Id}$, and the untwisted ones (i.e. those with $\sigma=\mathrm{Id}$ ). Twisted affine Kac-Moody algebras (resp. groups) can be described as subsets of untwisted ones. Hence it is sufficient to describe the regularity conditions for untwisted Kac-Moody algebras (resp. groups).

Let us mention several widely used regularity conditions. For the description of most of them we need only the analytic structure of the Lie group $G$. Examples are the groups of continuous loops $L G, k$-differentiable loops $L^{k} G^{\sigma}$, smooth loops $L^{\infty} G^{\sigma}$, real analytic or complex analytic loops $M G^{\sigma}$ resp. $A_{n} G^{\sigma}$ on $X \in\left\{\mathbb{C}^{*}, A_{n}:=\{z \in\right.$ $\left.\mathbb{C}\left|e^{-n} \leq\right| z \mid \leq e^{n}\right\}$ (for the last two cases to make sense, we need $G$ to be a complex Lie group).

In contrast the precise meaning of algebraic (or polynomial) loops into a Lie group is not clear a priori.

The algebraic loop (resp. Kac-Moody) group is the smallest group, we are interested in: We put

$$
L_{\mathrm{alg}} G^{\sigma}:=\{f \in L(G, \sigma) \mid \text { has a finite Fourier expansion }\},
$$

where the Fourier expansion is defined via the adjoint representation of $G$. If $G$ is complex, we can identify this group with a group of matrix-valued Laurent polynomials [PS86]. By construction, this group is isomorphic to the group $G\left(\mathbb{C}\left[t, t^{-1}\right]\right)$, the realization of the affine algebraic group scheme corresponding to the Lie group $G$ over the ring $\mathbb{C}\left[t, t^{-1}\right]$ (for the definition [AB08] or [Wat79]. Remark that $G\left(\mathbb{C}\left[t, t^{-1}\right]\right.$ ) is the group acting in a natural way on a twin building [Ron03], [Rém02].

Let us now investigate some relationships between these regularity conditions.

If $G$ is semisimple compact or complex, then $L_{\mathrm{alg}} G$ is dense in the group of continuous loops $L G$ [PS86], chapter 3.5. Hence ${\widehat{L_{\mathrm{alg}} G}}^{\sigma}$ is dense in $\widehat{L}(G, \sigma)$. Thus the Kac-Moody groups $\widehat{L}(G, \sigma)$ for $G$ semisimple are completions of the corresponding algebraic Kac-Moody groups ${\widehat{L_{a l g} G}}^{\sigma}$. Conversely ${\widehat{L_{a l g} G}}^{\sigma}$ is in a natural way a subgroup of $\widehat{L}(G, \sigma)$. Call a subgroup of $\widehat{L}(G, \sigma)$ which is abstractly isomorphic to the 
group of algebraic loops $L_{\mathrm{alg}} G^{\sigma}$ a quasi-algebraic subgroup — denoted ${\widehat{L_{\mathrm{qalg}} G}}^{\sigma}$ - of $\widehat{L}(G, \sigma)$.

${\widehat{L_{\mathrm{alg}} G}}^{\sigma}$ is clearly not the only quasi-algebraic subgroup of $\widehat{L}(G, \sigma)$. For example for any $f \in \widehat{L}(G, \sigma)$ the subgroup ${\widehat{L_{\text {alg }} G}}_{f}^{\sigma}:=f{\widehat{L_{\text {alg }} G}}^{\sigma} f^{-1}$ is quasi-algebraic.

We want to show that all quasi-algebraic groups are conjugate. To this end we need the following definition:

Definition 2.1. A group $\widehat{L}(G, \sigma)$ satisfies the conjugation property iff all tori of finite type are conjugate.

The conjugation property is satisfied for example by the groups $\widehat{M G}^{\sigma},{\widehat{A_{n} G}}^{\sigma}$ and their compact real forms by the following theorem:

Theorem 2.1. All tori of finite type in $\widehat{X G}^{\sigma}, X \in\left\{A_{n}, M\right\}$ are conjugate.

Proof. [Fre09].

Remark that the conjugation property is satisfied for most interesting regularity classes, among them the groups of $H^{1}$-Sobolev loops [Ter95], of $k$-times differentiable loops [Fre09] and of smooth loops [Pop05], [Fre09].

Theorem 2.2 (Algebraic subgroups). Let $G$ be a simple, simply connected, compact or complex Lie group. Suppose $\widehat{L}(G, \sigma)$ satisfies the conjugation property. Let ${\widehat{L_{q a l g} G}}^{\sigma} \subset \widehat{L}(G, \sigma)$ be a quasi-algebraic subgroup. Then there is $f \in \widehat{L}(G, \sigma)$ such that ${\widehat{L_{\text {qalg }} G}}^{\sigma}:=f{\widehat{L_{\text {alg }} G}}^{\sigma} f^{-1}$.

For the proof we use the following observation [PS86], proposition 5.2.5:

LEMMA 2.1. If $G$ is a simple, simply connected compact Lie group of rank $l$, then the $l+1$ subgroups $i_{\alpha}(S U(2))$ corresponding to the simple affine roots generate $\widehat{L_{\text {alg }} G}$.

Remember, that $\widetilde{L_{a l g} G}$ denotes the central extension of $L_{a l g} G$. If $G$ is not simply connected, then $L G$ is not connected. In this case the subgroups $i_{\alpha}(S U(2))$ generate the identity component $\left(\widetilde{L_{\text {alg }} G}\right)_{0}$. The proof of [PS86] generalizes to the case of twisted loop groups, as it relies only on the algebraic structure of generators and relations of the Kac-Moody algebra.

A similar result holds for complex Lie groups:

Lemma 2.2. If $G_{\mathbb{C}}$ is a simple, simply connected complex Lie group of rank l, then the $l+1$ subgroups $i_{\alpha}\left(S L_{2}(\mathbb{C})\right)$ corresponding to the simple affine roots generate $\widetilde{L_{a l g} G_{\mathbb{C}}}$.

This is the loop group version of the description of complex affine Kac-Moody groups as the amalgam of its $S L_{2}(\mathbb{C})$ subgroups [Cap09], [Rém02].

Hence a quasi-algebraic subgroup ${\widehat{L_{\text {qalg }} G}}^{\sigma}$ of a group $\widehat{L}(G, \sigma)$ is completely described by the choice of a maximal torus. An embedding $\varphi: \widehat{L a l g}^{\sigma} \longrightarrow \widehat{L}(G, \sigma)$ is determined by the choice of an isomorphism of a torus and the family of isomorphisms of the $S U(2)$ - resp. $S L_{2}(\mathbb{C})$-subgroups corresponding to the simple roots. Hence we get the following lemma:

Lemma 2.3. Let $G$ be a simple, simply connected compact or complex Lie group and let $\varphi_{i}:{\widehat{L_{a l g} G}}^{\sigma} \longrightarrow \widehat{L}(G, \sigma), i=\{1,2\}$ be two embeddings, let $\widehat{T}$ be a maximal torus in ${\widehat{L_{\text {alg }} G}}^{\sigma}$ and $i_{\alpha}(S U(2))$ the subgroups associated to the simple roots. 


$$
\text { If } \varphi_{1}(\widehat{T})=\varphi_{2}(\widehat{T}) \text { and } \varphi_{1}\left(i_{\alpha} S U(2)\right)=\varphi_{2}\left(i_{\alpha} S U(2)\right) \forall \alpha \text {, then } \varphi_{1}=\varphi_{2} \text {. }
$$

Proof. Two group isomorphisms are equivalent iff they coincide on a set of generators. By lemma 2.1 (for compact $G$ ) resp. 2.2 (for complex $G$ ), ${\widehat{L_{a l g} G}}^{\sigma}$ is generated by a torus and the $S U(2)$ - resp. $S L_{2}(\mathbb{C})$-subgroups associated to the simple roots. This proves the lemma.

We are now in a position to prove theorem 2.2:

Proof. Let $G_{i} \subset \widehat{L}(G, \sigma), i \in\{1,2\}$ be two quasi-algebraic subgroups of $\widehat{L}(G, \sigma)$. Choose two tori of finite type $\widehat{T}_{1} \subset G_{1}$ and $\widehat{T}_{2} \subset G_{2}$. By the conjugation property, there is $g \in \widehat{L}(G, \sigma)$ such that $\widehat{T}_{1}=g \widehat{T}_{2} g^{-1}$. Define $H_{2}:=g G_{2} g^{-1}$. The groups $G_{1}$ and $H_{2}$ share the maximal torus $\widehat{T}_{1}$. Hence they have the same root system with respect to $\widehat{T}_{1}$. Choose in both groups the same system of simple roots. The $S U(2)$ resp. $S L_{2}(\mathbb{C})$-subgroups associated to those simple roots are well defined. Hence they coincide. Thus by lemma $2.1, G_{1}$ and $H_{2}$ coincide. Thus $G_{1}$ and $G_{2}$ are conjugate.

Next we investigate Borel subgroups and parabolic subgroups.

Definition 2.2 (countably solvable subgroup). A subgroup $S \subset \widehat{L}(G, \sigma)$ is called countably solvable if the upper central series converges to the identity.

Definition 2.3. A Borel group of $\widehat{L}(G, \sigma)$ is a maximal connected, closed, countably solvable subgroup of $\widehat{L}(G, \sigma)$.

Other equivalent definitions are proposed in [Rém02] and [Kum02].

As usual, two Borel subgroups $B^{+}$and $B^{-}$in a Kac-Moody group $\widehat{L}(G, \sigma)$ are opposite, iff $B^{+} \cap B^{-} \simeq \widehat{T}$. Call $\widehat{N}$ the normalizer of $\widehat{T}$ and put $W=\widehat{N} / \widehat{T}$. $\widehat{N}$ and hence also $W$ are independent of the regularity of $\widehat{L}(G, \sigma)$.

Let $B^{+}$and $B^{-}$be two opposite Borel subgroups. Then they describe a unique quasi-algebraic subgroup $G\left(B^{+}, B^{-}\right) \subset \widehat{L}(G, \sigma)$ : We construct this group by taking the torus $\widehat{T}=B^{+} \cap B^{-}$and taking the group generated by $\widehat{T}$ and the simple root groups. This group has a twin $B N$-pair consisting of $\left(B^{+} \cap G\left(B^{+}, B^{-}\right), B^{-} \cap\right.$ $\left.G\left(B^{+}, B^{-}\right), N, W, S\right)$.

Hence, given a pair of opposite Borel subgroups, we get in each of the two Borel subgroup a Borel subgroup corresponding to the quasi-algebraic group $G\left(B^{+}, B^{-}\right)$. We call this Borel subgroup the algebraic kernel of $B^{\epsilon}(\epsilon \in \pm)$ with respect to $B^{-\epsilon}$.

More generally, let $B$ be a Borel group. As $B$ is a countably solvable group, we get a series of subgroups $B^{(i+1)}=\left[B^{(i)}, B^{(i)}\right]$. Those groups are well defined. But we don't get complementary subspaces. Those have to be chosen explicitly. We denote by $T^{(i)}$ a series of subspaces such that $B^{(i+1)} \simeq T^{(i)} \oplus B^{(i)}$.

$T^{(0)}$ is just a maximal torus of finite type. $T^{(0)}$ uniquely determines a set of root subgroups. Define $T^{(1)}$ to be the union of the root subgroups of simple roots and similarly for $T^{(i)}:=\left\{a b \mid a, b \in T^{(i-1)}\right\} \cap B^{(i+1)}, i>0$. From now on, by $T^{(i)}$ we will denote this set of subspaces.

Definition 2.4 ( $T$-algebraic subgroup). The T-algebraic subgroup $B_{\text {alg }}^{T}$ of a Borel subgroup $B$ with respect to a maximal torus $T$ is the subgroup of elements $f \subset B$ such that there exist some $n \in \mathbb{N}$ such that $f \subset \bigcup_{i=0}^{n} T^{(i)}$. 
LEMma 2.4. Let $G\left(B^{+}, B^{-}\right)$be a quasi-algebraic subgroup of $\widehat{L}(G, \sigma)$ and $T$ a torus in $G\left(B^{+}, B^{-}\right)$that is a complement to $B^{+,(1)}$ in $B^{+}$. Then

$$
B^{+} \cap G\left(B^{+}, B^{-}\right)=B_{a l g}^{+, T}
$$

Proof. Let $T$ be a torus in $G\left(B^{+}, B^{-}\right)$as described in the lemma. We claim: There is a Borel subgroup $\widetilde{B}^{-}$such that $T=B^{+} \cap \widetilde{B}^{-}$and $G\left(B^{+}, \widetilde{B}^{-}\right)=G\left(B^{+}, B^{-}\right)$. Then the lemma follows from the definitions. Hence we are left with proving our claim: We construct $\widetilde{B}^{-}$explicitly to be the completion of the negative root subgroups.

LEMma 2.5. All positive (resp. negative) Borel subgroups $B^{+}$(resp. $B^{-}$) of $\widehat{L}(G, \sigma)$ are conjugate.

In the case of $G L(n, \mathbb{C})$, the proof proceeds as follows: Each Borel subgroup fixes a unique maximal flag. Hence the result follows as $G L(n, \mathbb{C})$ is transitive on maximal flags. If $G \subset G L(n, \mathbb{C})$ the proof follows using the embedding.

Proof. The proof studies the action of $L(G, \sigma)$ on a suitable vector space and shows that it is transitive on spaces of periodic flags. For details [PS86], section 7 and 8 and [Fre11a].

We summarize our results: Each pair of two opposite Borel subgroups $B^{ \pm}$defines exactly one maximal torus and hence a quasi-algebraic subgroup of $\widehat{L}(G, \sigma)$. We denote this group $G\left(B^{+}, B^{-}\right)$. Quasi algebraic subgroups, such that a given Borel group $B$ is the completion of an algebraic Borel subgroup $B_{\text {alg }}$, contain a torus which is a complement to $B^{(1)}$ in $B$.

3. Summary of the algebraic theory. In this section we gather some basic results about affine twin buildings for minimal Kac-Moody groups. Two references for this section are [AB08] and [Rém02].

Definition 3.1 (Twin BN-pair). Let $\widehat{L_{\text {alg }} G_{\mathbb{C}}}$ be a complex Kac-Moody group. Let $\widehat{B}^{+}$and $\widehat{B}^{-}$be opposite Borel subgroups $T=\widehat{B}^{+} \cap \widehat{B}^{-}, N$ the stabilizer of $T, W$ the Weyl group and $S$ a set of generators for $W$. The quintupel $\left(\widehat{B}^{+}, \widehat{B}^{-}, N, W, S\right)$ is a twin $B N$-pair for $\widehat{L_{\text {alg }} G_{\mathbb{C}}}$ iff:

1. $\left(\widehat{B}^{+}, N, W, S\right)$ is a $B N$-pair (called $\left.B^{+} N\right)$,

2. $\left(\widehat{B}^{-}, N, W, S\right)$ is a $B N$-pair (called $\left.B^{-} N\right)$,

3. $B^{+} N$ and $B^{-} N$ are compatible, i.e.

(a) If $l(w s)<l(w)$ then $\widehat{B}^{\epsilon} w \widehat{B}^{-\epsilon} s \widehat{B}^{-\epsilon}=\widehat{B}^{\epsilon} w s \widehat{B}^{-\epsilon}$ for $\epsilon \in\{+,-\}, w \in$ $W, s \in S$,

(b) $\widehat{B}^{+} s \cap \widehat{B}^{-}=\emptyset \forall s \in S$.

We use the notation $w(f)$ (resp. in case of ambiguity: $w^{\epsilon}(f), w^{ \pm}(f), w^{\mp}(f)$ ) to denote the class of $f$ in the corresponding Bruhat decomposition. The existence of the twin $B N$-pairs yields Bruhat decompositions for $\widehat{L_{\text {alg }} G_{\mathbb{C}}}$ similar to the finite dimensional case:

THEOREM 3.1 (Bruhat decomposition). Let $\widehat{L_{a l g} G}$ be an affine algebraic KacMoody group with affine Weyl group $W_{\text {aff. }}$. Let furthermore $B^{ \pm}$denote a positive (resp. negative) Borel group. There are decompositions

$$
\widehat{L_{a l g} G}=\coprod_{w \in W_{a f f}} \widehat{B}^{+} w \widehat{B}^{+}=\coprod_{w \in W_{a f f}} \widehat{B}^{-} w \widehat{B}^{-} .
$$


THEOREM 3.2 (Bruhat twin decomposition). Let $\widehat{L_{\text {alg }} G}$ be an affine algebraic Kac-Moody group with affine Weyl group $W_{\text {aff. }}$. Let furthermore $B^{+}$denote a positive Borel subgroup and $B^{-}$its opposite negative Borel group. There are decompositions

$$
\widehat{L_{a l g} G}=\coprod_{w \in W_{a f f}} \widehat{B}^{\epsilon} w \widehat{B}^{-\epsilon} \quad \text { for } \epsilon \in\{+,-\} .
$$

For proofs of those results see any book about Kac-Moody groups, i.e. [Rém02], chapter 1. What we call in this work Bruhat twin decomposition is sometimes called Birkhoff decomposition.

Note that the Bruhat decompositions and the Bruhat twin decompositions are defined on the whole group $\widehat{L_{a l g} G}$. For the associated buildings, this translates into the fact that any two chambers in $\mathfrak{B}^{+}$resp. $\mathfrak{B}^{-}$have a well-defined Weyl distance and a well-defined Weyl codistance (compare definition 3.4).

The same results hold for $L_{a l g} G$.

Definition 3.2 (BN-flip). An involution $\varphi$ of a Kac-Moody group is called a $B N$-flip iff

1. $\varphi^{2}=1$,

2. $\varphi\left(\widehat{B}^{+}\right)=\widehat{B}^{-}$,

3. $\varphi$ centralizes the Weyl group.

A $B N$-flip swaps the two $B N$-pairs. The existence of a $B N$-flip is a sign of symmetry of the group structure, which can be lost for non-algebraic Kac-Moody groups.

Similarly to the two conjugacy classes of Borel subgroups, the set of affine parabolic subgroups breaks up into two classes. The first one consists of affine parabolic subgroups containing a conjugate of $\widehat{B}^{+}$, the second one of those containing a conjugate of $\widehat{B}^{-}$. The two sets of parabolic subgroups admit a partial order relation exactly as in the finite dimensional case. To this complex, one can associate a simplicial complex, which has the structure of an affine Tits building. The apartments are Coxeter complexes for $W_{\text {aff }}$.

A big difference between affine buildings and spherical ones is that chambers in affine buildings do not have opposite chambers (recall that two chambers are called opposite if their Weyl distance is maximal, which is not possible in an affine Weyl group). As the existence of opposite chambers is a necessary ingredient for various structure results, this is a serious detriment. The most important consequence of the existence of opposite chambers for spherical buildings is the following theorem:

THEOREM 3.3. In a spherical building, apartments are exactly the convex hulls of a pair of opposite chambers.

Proof. [AB08].

This theorem implies the corollary:

COROLLARY 3.1. The apartment system in a spherical building is unique.

In contrast affine buildings have various different apartment systems, reflecting the different completions of the associated Kac-Moody groups.

So there is a need for a version of the concept of opposite chambers for affine buildings, which should then lead to a generalization of theorem 3.3. It is clear that an opposite chamber cannot be in the same building, as it would have a Weyl distance 
of maximal length. Thus the solution lies in a twinning of the two buildings associated to the two $B N$-pairs. The resulting object, called a twin building, behaves in many respects like a spherical building.

We quote the $W$-metric definition of a building from the monograph [AB08], chapter 5 .

Definition 3.3 (building). A building of type $(W, S)$ is a pair $(\mathcal{C}, \delta)$ consisting of a nonempty set $\mathcal{C}$ whose elements are called chambers together with a map $\delta$ : $\mathcal{C} \times \mathcal{C} \longrightarrow W$, called the Weyl distance function, such that for all $C, D \in \mathcal{C}$ the following conditions hold:

1. $\delta(C, D)=1$ iff $C=D$.

2. If $\delta(C, D)=w$ and $C^{\prime} \in \mathcal{C}$ satisfies $\delta\left(C^{\prime}, C\right)=s \in S$ then $\delta\left(C^{\prime}, D\right)=s w$ or $w$. If in addition $l(s w)=l(w)+1$ then $\delta\left(C^{\prime}, D\right)=s w$.

3. If $\delta(C, D)=w$ then for any $s \in S$ there is a chamber $C^{\prime} \subset \mathcal{C}$, such that $\delta\left(C^{\prime}, C\right)=s$ and $\delta\left(C^{\prime}, D\right)=s w$.

This definition coincides with the classical definition of a building as a simplicial complex. For a proof $[\mathrm{AB} 08]$. The construction of the apartments is somewhat involved.

Definition 3.4 (Twin building). A twin building of type $(W, S)$ is a quintuple $\left(\mathcal{C}^{+}, \mathcal{C}^{-}, \delta^{+}, \delta^{-}, \delta^{*}\right)$ such that

1. $\left(\mathcal{C}^{+}, \delta^{+}\right)$is a building of type $(W, S)$,

2. $\left(\mathcal{C}^{-}, \delta^{-}\right)$is a building of type $(W, S)$,

3. $\delta^{*}$ is a codistance, i.e. for $X \in \mathfrak{B}^{\epsilon}$ and $Y, Z \in \mathfrak{B}^{-\epsilon}$,

a) $\delta^{*}(X, Y)=\delta^{*}(Y, X)^{-1}$,

b) $\delta^{*}(X, Y)=w, \delta(Y, Z)=s \in S$ and $l(w s)=l(w)-1$, then $\delta^{*}(X, Z)=$ $w s$,

c) $\delta^{*}(X, Y)=w$ and $s \in S$. Then there is $Z \in \mathfrak{B}^{-}$such that $\delta^{*}(Y, Z)=s$ and $\delta^{*}(X, Z)=w s$.

Definition 3.5. $X$ and $Y$ are called opposite iff $\delta^{*}(X, Y)=1$.

For a pair of affine buildings the twinning is in general not uniquely determined. There are uncountable many non-isomorphic twinnings. In the case of rank 1-buildings - that is trees -, a universal twin building has been constructed by Mark Ronan and Jacques Tits [RT94], [RT99]. For more general classes of buildings this is an open problem.

A twinning can be described via twin apartments. Here, we have again the result that the system of twin apartments is well defined. Furthermore twin apartments are the convex hulls of opposite chambers [AR98].

4. Twin cities. In this section we construct cities associated to simple geometric affine Kac-Moody algebras $\widehat{L}(\mathfrak{g}, \sigma)$ and their Kac-Moody groups $\widehat{L}(G, \sigma)$.

There are two major obstacles:

1. Twin buildings correspond only to the subgroup of algebraic loops, or taking into account that the subgroup of algebraic loops is just one distinguished element in the conjugacy class of quasi-algebraic groups.

2. The completions of Kac-Moody groups do not act properly on twin buildings.

To resolve those problems, we define geometric $B N$-pairs and their associated cities. Those cities are chamber complexes such that $\mathfrak{B}^{+}$and $\mathfrak{B}^{-}$each consist of an infinite number of connected components, each of which is an affine building, such that each pair consisting of a building $\Delta^{+}$in $\mathfrak{B}^{+}$and a building $\Delta^{-}$in $\mathfrak{B}^{-}$is a twin building in the classical algebraic sense. 


\subsection{Geometric $B N$-pairs.}

Definition 4.1 (Geometric $B N$-pair for $\widehat{L}(G, \sigma)$ ).

Let $\widehat{L}(G, \sigma)$ be an affine Kac-Moody group. $\left(B^{+}, B^{-}, N, W, S\right)$ is a twin BN-pair for $\widehat{L}(G, \sigma)$ iff there are subgroups $\widehat{L}(G, \sigma)^{+}$and $\widehat{L}(G, \sigma)^{-}$of $\widehat{L}(G, \sigma)$ such that $\widehat{L}(G, \sigma)=\left\langle\widehat{L}(G, \sigma)^{+}, \widehat{L}(G, \sigma)^{-}\right\rangle$is subject to the following axioms:

1. $\left(B^{+}, N, W, S\right)$ is a $B N$-pair for $\widehat{L}(G, \sigma)^{+}\left(\right.$called $\left.B^{+} N\right)$,

2. $\left(B^{-}, N, W, S\right)$ is a $B N$-pair for $\widehat{L}(G, \sigma)^{-}$(called $\left.B^{-} N\right)$,

3. $\left(B^{+} \cap \widehat{L}(G, \sigma)^{-}, B^{-} \cap \widehat{L}(G, \sigma)^{+}, N, W, S\right)$ is a twin $B N$-pair for $\widehat{L}(G, \sigma)^{+} \cap$ $\widehat{L}(G, \sigma)^{-}$.

The subgroups $\widehat{L}(G, \sigma)^{+}$and $\widehat{L}(G, \sigma)^{-}$of $\widehat{L}(G, \sigma)$ depend on the choice of $B^{+}$ and $B^{-}$. A choice of a different subgroup $B^{+^{\prime}}$ (resp. $B^{-^{\prime}}$ ) gives the same subgroup $\widehat{L}(G, \sigma)^{+}$(resp. $\left.\widehat{L}(G, \sigma)^{-}\right)$of $\widehat{L}(G, \sigma)$ if $B^{+^{\prime}} \subset \widehat{L}(G, \sigma)^{+}$(resp. $B^{-^{\prime}} \subset \widehat{L}(G, \sigma)$ if $\left.B^{-^{\prime}} \subset \widehat{L}(G, \sigma)^{-}\right)$. For all positive (resp. negative) Borel subgroups the positive (resp. negative) subgroups $\widehat{L}(G, \sigma)^{+}$resp. $\widehat{L}(G, \sigma)^{-}$are conjugate. Hence without loss of generality we can think of $B^{ \pm}$to be the standard positive (resp. negative) affine Borel subgroup. The groups $\widehat{L}(G, \sigma)^{ \pm}$- called the standard positive (resp. negative) subgroups - are then characterized by the condition that 0 (resp. $\infty$ ) is of finite order for all elements.

Remark 4.1. For an algebraic Kac-Moody group a geometric BN-pair coincides with a $B N$-pair. Hence we get $\widehat{L}(G, \sigma)^{+}=\widehat{L}(G, \sigma)^{-}=\widehat{L}(G, \sigma)$.

We use the equivalent definitions for the loop groups $L(G, \sigma)$.

The $W$-metric description of buildings shows that the structure of a twin building is intimately related to the Bruhat and the Bruhat twin decomposition. For completed Kac-Moody groups, those decompositions need no longer be globally defined. This new feature is crucial for the disconnected structure of cities.

LEMMA 4.1.

1. The groups $L(G, \sigma)^{+}$(resp. $\left.L(G, \sigma)^{-}\right)$have a positive (resp. negative) Bruhat decomposition and a Bruhat twin decomposition.

2. The group $L(G, \sigma)$ has a Bruhat twin decomposition but no Bruhat decomposition.

Proof. The Bruhat decomposition in the first part follows by definition, the Bruhat twin decomposition by restriction and the second part. The second part is a restatement of the decomposition results in chapter 8 of [PS86].

Compare also similar decomposition results stated in [Tit84].

TheOrem 4.1 (Bruhat decomposition). Let $\widehat{L}(G, \sigma)$ be an affine Kac-Moody group with affine Weyl group $W_{\text {aff. }}$. Let furthermore $B^{ \pm}$denote a positive (resp. negative) Borel group. There are decompositions

$$
\widehat{L}(G, \sigma)^{+}=\coprod_{w \in W_{\text {aff }}} B^{+} w B^{+}
$$

and

$$
\widehat{L}(G, \sigma)^{-}=\coprod_{w \in W_{\text {aff }}} B^{-} w B^{-}
$$


Proof. This is a consequence of lemma 4.1.

THEOREM 4.2 (Bruhat twin decomposition). Let $\widehat{L}(G, \sigma)$ be an affine algebraic Kac-Moody group with affine Weyl group $W_{\text {aff. }}$ Let furthermore $B^{ \pm}$denote a positive and its opposite negative Borel group. There are two decompositions

$$
\widehat{L}(G, \sigma)=\coprod_{w \in W_{a f f}} B^{ \pm} w B^{\mp} .
$$

REMARK 4.2. Note that the Bruhat twin decomposition is defined on the whole group $\widehat{L}(G, \sigma)$. For cities, this translates into the fact that any two chambers in $\mathfrak{B}^{+}$ resp. $\mathfrak{B}^{-}$have a well-defined Weyl codistance - see subsection 4.2. In contrast, Bruhat decompositions are only defined for the subgroups $\widehat{L}(G, \sigma)^{ \pm}$. This translates into the fact that there are positive (resp. negative) chambers without a well-defined Weyl distance, hence that the buildings will be disconnected.

ExAmPLE 4.1. Shrawan Kumar studies formal completions of Kac-Moody groups and Kac-Moody algebras. Those groups complete in only "one direction"/ i.e. with respect to one of the two opposite BN-pairs. Similarly in the setting of affine KacMoody groups of holomorphic loops we could use holomorphic functions with finite principal part. There is an associated twin BN-pair; the positive Borel subgroups are completed affine Borel subgroups while the negative ones are the algebraic affine Borel subgroups. Thus for a geometric twin $B N$-pair we have to use: $\widehat{L}(G, \sigma)^{+}=$ $\widehat{L}(G, \sigma)$ and $\widehat{L}(G, \sigma)^{-}=\widehat{L}$ alg $^{\sigma}$ (Kumar studies only the affine building associated to $B N^{+}[$Kum02]).

Definition 4.2. An involution $\varphi: \widehat{L}(G, \sigma) \longrightarrow \widehat{L}(G, \sigma)$ is called a $B N$-flip iff

1. $\varphi^{2}=1$,

2. $\varphi\left(B^{+}\right)=B^{-}$,

3. $\varphi$ centralizes $W$.

Definition 4.3. A geometric twin BN-pair is called symmetric iff it has a BN-flip.

ExAMPLE 4.2. For a twin BN-pair to be symmetric we need that $B^{+}$and $B^{-}$are isomorphic groups. More precisely this means that the completion has to be symmetric in both directions. Thus the groups of example 4.1 have non symmetric geometric twin $B N$-pairs.

EXAMPLE 4.3. An algebraic affine twin BN-pair is symmetric.

EXAMPLE 4.4. The geometric BN-pair associated to any group $\widehat{L}(G, \sigma)$ is symmetric.

LEMmA 4.2. The intersection $\widehat{L}(G, \sigma)^{0}$ of $\widehat{L}(G, \sigma)^{+}$with $\widehat{L}(G, \sigma)^{-}$is a quasialgebraic subgroup. For the standard affine Borel subgroups, it is the algebraic KacMoody group

$$
\widehat{L}(G, \sigma)^{0} \simeq{\widehat{L_{a l g} G}}^{\sigma}
$$

Proof. $\widehat{L}$ alg $G^{\sigma}$ is the maximal subgroup of $\widehat{L}(G, \sigma)$ having both Bruhat decompositions. 
4.2. Combinatorics of cities. We now define a twin city using the $W$-metric description:

Definition 4.4 (Twin City). Let $\widehat{L}(G, \sigma)$ be an affine Kac-Moody group with a geometric $B N$-pair and a Weyl group $W_{\text {aff. }}$. Define $\mathcal{C}^{+}:=\widehat{L}(G, \sigma) / B^{+}$and $\mathcal{C}^{-}:=$ $\widehat{L}(G, \sigma) / B^{-}$.

1. The distance $\delta^{\epsilon}: \mathcal{C}^{\epsilon} \times \mathcal{C}^{\epsilon} \longrightarrow W_{a f f}, \epsilon \in\{+,-\}$ is defined as usual via the Bruhat decompositions: $\delta^{\epsilon}\left(g B^{\epsilon}, f B^{\epsilon}\right)=w\left(g^{-1} f\right)$ if $g^{-1} f \in \widehat{L}(G, \sigma)^{\epsilon}$. Otherwise it is $\infty$.

2. The codistance $\delta^{*}: \mathcal{C}^{+} \times \mathcal{C}^{-} \cup \mathcal{C}^{-} \times \mathcal{C}^{+} \longrightarrow W$ is defined as usual via the Bruhat twin decompositions: $\delta^{*}\left(g B^{-}, f B^{+}\right)=w^{\mp}\left(g^{-1} f\right) \quad\left(\right.$ resp. $\delta^{*}\left(g B^{+}, f B^{-}\right)=$ $\left.w^{ \pm}\left(g^{-1} f\right)\right)$.

The elements of $\mathcal{C}^{ \pm}$are called the positive (resp. negative) chambers of the twin city. The building is denoted $\mathfrak{B}=\mathfrak{B}^{+} \cup \mathfrak{B}^{-}$. One can define a simplicial complex realization in the usual way. We define connected components in $\mathfrak{B}^{ \pm}$in the following way: Two elements $\left\{c_{1}, c_{2}\right\} \in \mathfrak{B}^{ \pm}$are in the same connected component iff $\delta^{ \pm}\left(c_{1}, c_{2}\right) \in$ $W_{\text {aff. }}$. We will check that this is an equivalence relation. Denote the set of connected components by $\pi_{0}(\mathfrak{B})$ resp. $\pi_{0}\left(\mathfrak{B}^{ \pm}\right)$.

Remark 4.3. Let $\widehat{L}(G, \sigma)$ be an algebraic affine Kac-Moody group. Then each city consists of exactly one building: Hence the twin city coincides with the twin building.

Lemma 4.3 (Properties of a twin city).

1. The connected components of $\mathfrak{B}^{\epsilon}$ are affine buildings of type $(W, S)$.

2. Each pair consisting of one affine building in $\mathfrak{B}^{+}$and one in $\mathfrak{B}^{-}$is a twin building of type $(W, S)$.

3. The connected components of $\mathfrak{B}^{\epsilon}$ are indexed by elements in $\widehat{L}(G, \sigma) / \widehat{L}(G, \sigma)^{\epsilon}$.

Proof.

1. Call two elements equivalent iff they are in the same connected component. This relation is clearly symmetric and self-reflexive. To prove transitivity, let $f B^{ \pm}, g B^{ \pm}$and $h B^{ \pm}$be such that there are $w_{f g}, w_{g h} \in W$ such that $f^{-1} g \in B^{ \pm} w_{f g} B^{ \pm}$and $g^{-1} h \in B^{ \pm} w_{g h} B^{ \pm}$. Then $f^{-1} h=f^{-1} g g^{-1} h \in$ $B^{ \pm} w_{f g} B^{ \pm} w_{g h} B^{ \pm}$; hence the distance is in $W$ and thus finite. Connected components are exactly subsets with finite codistance. We have to check that each connected component fulfills the metric definition of a building.

(a) If $\delta\left(f B^{\epsilon}, g B^{\epsilon}\right)=w$ - hence there are $b_{1}, b_{2} \in B^{\epsilon}$ such that $f^{-1} g=$ $b_{1} w b_{2}$ 一, then $g^{-1} f=b_{2}^{-1} w^{-1} b_{1}^{-1}$. Hence $\delta\left(g B^{\epsilon}, f B^{\epsilon}\right)=w^{-1}$.

(b) If $\delta\left(f B^{\epsilon}, g B^{\epsilon}\right)=w$ and $\delta\left(f^{\prime} B^{\epsilon}, f B^{\epsilon}\right)=s$, then $\delta\left(f^{\prime} B^{\epsilon}, g B^{\epsilon}\right)=$ $w\left(f^{\prime}-1 g\right)=w\left(f^{\prime-1} f f^{-1} g\right) \subset w\left(f^{\prime}-1 f\right) w\left(f^{-1} g\right) \cup w\left(f^{-1} g\right) \in\{s w, w\}$. If $l(s w)=l(w)+1$, then $\delta\left(f^{\prime} B^{\epsilon}, g B^{\epsilon}\right)=s w$.

(c) Let $\delta\left(f B^{\epsilon}, g B^{\epsilon}\right)=w$ and denote by $C_{s}$ be the $s$-panel containing $f B^{\epsilon}$. There are two possibilities:

i. Either $w$ has a representation such that $w=s w^{\prime}$ and $l\left(s w^{\prime}\right)>l\left(w^{\prime}\right)$ - i.e. the first letter of any reduced word representing $w^{\prime}$ in the generators $s_{i}$ is not $s$. As the last letter of $w$ is $s$, the last chamber of the gallery connecting $f B^{\epsilon}$ and $g B^{\epsilon}$, denoted $f^{\prime} B^{\epsilon}$, is contained in the $s$-panel $C_{s}$. Hence $\delta\left(f^{\prime} B^{\epsilon}, g B^{\epsilon}\right)=w^{\prime}=s w$. 
ii. If $w$ has no representation of the form $w=s w^{\prime}$ such that $l\left(s w^{\prime}\right)>l\left(w^{\prime}\right)$, then any chamber $f B^{\epsilon}$ in the panel $C(s)$ satisfies $\delta\left(f^{\prime} B^{\epsilon}, g B^{\epsilon}\right)=s w$.

2. Each pair consisting of one connected component in $\mathfrak{B}^{+}$and one in $\mathfrak{B}^{-}$ fulfills the axioms of definition 3.4. As the Bruhat decomposition is defined on $\widehat{L}(G, \sigma)$, the codistance is defined between arbitrary chambers in $\mathfrak{B}^{\epsilon}$ resp. $\mathfrak{B}^{-\epsilon}$.

3. $\widehat{L}(G, \sigma)$ has a decomposition into subsets of the form $\widehat{L}(G, \sigma)^{\epsilon}$. Those subsets are indexed with elements in $\widehat{L}(G, \sigma) / \widehat{L}(G, \sigma)^{\epsilon}$. The class corresponding to the neutral element is $\widehat{L}(G, \sigma)^{\epsilon} \subset \widehat{L}(G, \sigma)$. Thus it corresponds to a connected component and a building of type $(W, S)$. The result follows via translation by elements in $\widehat{L}(G, \sigma) / \widehat{L}(G, \sigma)^{\epsilon}$ : a connected component of $\mathfrak{B}^{\epsilon}$ containing $f B^{\epsilon}$ consists of all elements $f \widehat{L}(G, \sigma)^{\epsilon} B^{\epsilon}$ as $\delta\left(f h B^{\epsilon}, f h^{\prime} B^{\epsilon}\right)=w\left((f h)^{-1} f h^{\prime}\right)=$ $w\left(h^{-1} f^{-1} f h^{\prime}\right)=w\left(h^{-1} h^{\prime}\right) \in W$ as $h, h^{\prime} \in \widehat{L}(G, \sigma)$.

Definition 4.5. A twin city $\mathfrak{B}$ is symmetric iff there is a simplicial complex involution $\varphi_{\mathfrak{B}}: \mathfrak{B} \longrightarrow \mathfrak{B}$ such that $\varphi_{\mathfrak{B}}\left(\mathfrak{B}^{\epsilon}\right)=\mathfrak{B}^{-\epsilon}$.

Lemma 4.4. A twin city is symmetric iff its geometric BN-pair is symmetric.

Proof. The $B N$-pair involution induces a building involution.

4.3. Group actions on the twin city. This section studies the action of $\widehat{L}(G, \sigma)$ on the twin city associated to it.

We recall the special case of an algebraic Kac-Moody group: Borel subgroups in an algebraic Kac-Moody group are exactly the stabilizers of chambers while parabolic subgroups are the stabilizers of simplices. Furthermore the action is isometric with respect to the Weyl distance.

LEMMA 4.5 (Action of $\widehat{L}(G, \sigma)$ ).

1. The action of $\widehat{L}(G, \sigma)$ on $\mathfrak{B}$ by left multiplication is isometric.

2. The Borel subgroups are exactly the stabilizers of the chambers, the parabolic subgroups are the stabilizers of all simplices.

3. $\widehat{L}(G, \sigma)^{\epsilon}$ acts on the identity component $\Delta_{0}^{\epsilon} \subset \mathfrak{B}^{\epsilon}$ by isometries.

4. Let $\Delta_{1}^{+} \cup \Delta_{1}^{-}$be an arbitrary twin building in $\mathfrak{B}$. Suppose $D^{ \pm}$are two opposite Borel subgroup stabilizing simplices in $\Delta_{0}^{ \pm}$. The group $G\left(D^{+}, D^{-}\right)$acts on $\Delta_{0}^{+} \cup \Delta_{0}^{-}$by isometries.

5. Let $f B^{\epsilon}$ and $g B^{\epsilon}$ be two chambers in the same connected component of $\mathfrak{B}^{\epsilon}$, and $h, h^{\prime} \in \widehat{L}(G, \sigma)^{-\epsilon}$. The left translates $h f B^{-\epsilon}$ and $h^{\prime} g B^{-\epsilon}$ are in the same connected component iff $f^{-1} h^{-1} h^{\prime} g \in \widehat{L}(G, \sigma)^{\epsilon}$.

Proof.

1. $G$ acts isometrically on a twin building if the action on both parts preserves the distances and the codistance [AB08], section 6.3.1. Hence the first assertion follows from the definition of $\mathcal{C}^{ \pm}$as coset spaces of $\widehat{L}(G, \sigma)$ and a direct check:

$$
\delta\left(h f B^{ \pm \epsilon}, h g B^{ \pm \epsilon}\right)=w\left(f^{-1} h^{-1} h g\right)=w\left(f^{-1} g\right)=\delta\left(f B^{ \pm \epsilon}, g B^{ \pm \epsilon}\right) .
$$

2. The chamber corresponding to $f B^{\epsilon}$ is stabilized by the Borel subgroup $B_{f}^{\epsilon}:=f B^{\epsilon} f^{-1}$. The converse follows as each Borel subgroup is conjugate to a standard one. Analogous for the parabolic subgroups. 
3. The identity component is described by $\widehat{L}(G, \sigma)^{\epsilon} B^{\epsilon}$. Hence it is preserved by left multiplication of $\widehat{L}(G, \sigma)^{\epsilon}$ - thus the action is well defined and isometric by the first statement.

4. The group $\widehat{L}(G, \sigma)^{\epsilon}=B^{\epsilon} W B^{\epsilon}$ acts by the last statement on $\Delta_{0}^{\epsilon}$ by isometries. Hence $G\left(B^{+}, B^{-}\right)=\widehat{L}(G, \sigma)^{+} \cap \widehat{L}(G, \sigma)^{-}$acts on $\Delta_{0}^{+} \cup \Delta_{0}^{-}$by isometries. Let $f B^{+} \in \Delta_{1}^{+}$and $g B^{-} \in \Delta_{1}^{-}$be the chambers stabilized by $D^{ \pm}$. Then $D^{+}=f B^{+} f^{-1}$ and $D^{-}=g B^{-} g^{-1}$. By theorem 2.2 there is some $h \in \widehat{L}(G, \sigma)$ such that $G\left(D^{+}, D^{-}\right)=h G\left(B^{+}, B^{-}\right) h^{-1}$. The groups $B^{\prime \pm}=h B^{ \pm} h^{-1}$ are Borel subgroups in $G\left(D^{+}, D^{-}\right)$. As all positive resp. negative Borel subgroups in $G\left(D^{+}, G^{-}\right)$are conjugate in $G\left(D^{+}, G^{-}\right)$, there are elements $f^{\prime}$ and $g^{\prime}$ in $G\left(D^{+}, G^{-}\right)$such that $D^{+}=f^{\prime} B^{\prime+} f^{\prime-1}=f^{\prime} h B^{+} h^{-1} f^{\prime-1}$ and $D^{-}=$ $g^{\prime} B^{\prime-} g^{-1}=g^{\prime} h B^{-} h^{-1} g^{\prime-1}$. Hence $f=f^{\prime} h b_{f}$ with $b_{f} \in B^{+}$and $g=g^{\prime} h b_{g}$ with $b_{g} \in B^{-}$.

Now we can prove that $\Delta_{1}^{+}$is invariant under $G\left(D^{+}, D^{-}\right)$: For $k \in$ $G\left(D^{+}, D^{-}\right)$we define $k_{0}=h^{-1} k h$ (hence $\left.k_{0} \in G\left(B^{+}, B^{-}\right) \subset \widehat{L}(G, \sigma)^{+}\right)$:

$$
\begin{aligned}
\delta\left(k f B^{+}, f B^{+}\right) & =w\left(f^{-1} k^{-1} f\right)= \\
& =w\left(f^{-1} h k_{0}^{-1} h^{-1} f\right)= \\
& =w\left(b_{f} h^{-1} f^{\prime} h k_{0}^{-1} f^{\prime} h b_{f}\right) .
\end{aligned}
$$

As $b_{f} \in B^{+} \subset \widehat{L}(G, \sigma)^{+}, h^{-1} f^{\prime-1} h \in G\left(B^{+}, B^{-}\right) \subset \widehat{L}(G, \sigma)^{+}, k_{0} \in$ $G\left(B^{+}, B^{-}\right) \subset \widehat{L}(G, \sigma)^{+}$, we find that $w\left(b_{f} h^{-1} f^{\prime} h k_{0}^{-1} f^{\prime} h b_{f}\right) \in W$. Hence $G\left(D^{+}, D^{-}\right)$preserves $\Delta_{1}^{+}$. Analogously we conclude for $\Delta_{1}^{-}$. This proves the claim.

5. $f^{-1} h^{-1} h^{\prime} g \in \widehat{L}(G, \sigma)^{\epsilon}$ is equivalent to $\delta^{\epsilon}\left(h f B^{-\epsilon}, h^{\prime} g B^{-\epsilon}\right) \in W_{\text {aff. }}$.

As quasi-algebraic subgroups are in bijection with algebraic twin buildings in $\mathfrak{B}$, we give a geometric characterization of them.

TheOREM 4.3. Let $H \subset \widehat{L}(G, I d)$ be a subgroup conjugate to $G$ and $\mathfrak{h}$ its Lie algebra. Then the group

$$
L_{H} G:=\left\{e^{t X_{1}} e^{t Y_{1}} \ldots e^{t X_{n}} e^{t Y_{n}} g \mid g \in H, X_{i}, Y_{i} \in \mathfrak{h}, e^{t X_{i}} e^{t Y_{i}}=e\right\}
$$

is quasi-algebraic. Conversely for each quasi-algebraic subgroup $\widehat{L_{q a l g} G}$ there are $H$ and $\mathfrak{h}$ such that $\widehat{L_{\text {qalg }} G}$ is of this form.

Before describing the proof, let us note as a corollary an application: We give a characterization of the connected components of $\mathfrak{B}^{\epsilon}$.

Corollary 4.1. Two simplices $\bar{f} B^{\epsilon}$ and $\bar{g} B^{\epsilon} \in \mathfrak{B}^{\epsilon}$ are contained in the same connected component $\Delta_{1}^{\epsilon}$ of $\mathfrak{B}^{\epsilon}$ iff there are representatives $f, g \in \widehat{L}(G, \sigma)$, $\left\{X_{1}, \ldots, X_{n}, Y_{1}, \ldots, Y_{n} \in \mathfrak{h} \mid e^{t X_{i}} e^{t Y_{i}}=e\right\}$ where $\mathfrak{g} \simeq \mathfrak{h} \subset \widehat{L}(\mathfrak{g}, \sigma)$ and a constant $c$ such that $f B^{\epsilon}=\bar{f} B^{\epsilon} g B^{\epsilon}=\bar{g} B^{\epsilon}$ and $f(t)=e^{t X_{1}} e^{t Y_{1}} \ldots e^{t X_{n}} e^{t Y_{n}} c \cdot g(t)$.

Proof. [Proof of corollary 4.1] Choose a quasi-algebraic subgroup $G\left(\Delta_{1}^{\epsilon}\right)$ acting transitively on $\Delta_{1}^{\epsilon}$. Choose $g$ to be an arbitrary representative of $\bar{g}$. Then there is $f^{\prime} \in G\left(\Delta_{1}\right)$ such that $f^{\prime} g$ is a representative for $\bar{f}$. Put $f:=f^{\prime} g$, choose an embedding $\varphi: \widehat{L_{\text {alg }} \mathfrak{g}} \longrightarrow G\left(\Delta_{1}^{\epsilon}\right)$ and put $\mathfrak{h}=\varphi(\mathfrak{g})$. Now the corollary follows from theorem 4.3. $\mathrm{u}$ 
The theorem is a consequence of the following lemma, due to Ernst Heintze, which is the special case for $H:=G$.

Lemma 4.6 (Characterization of $L_{a l g} G$ ).

$$
L_{\text {alg }} G:=\left\{e^{t X_{1}} e^{t Y_{1}} \ldots e^{t X_{n}} e^{t Y_{n}} g \mid g \in G, X_{i}, Y_{i} \in \mathfrak{g}, e^{t X_{i}} e^{t Y_{i}}=e\right\} .
$$

Proof of theorem 4.3.

1. Choose $k \in L(G, \sigma)$ such that $H=k G k^{-1}$. Then $\mathfrak{h}=k \mathfrak{g} k^{-1}$. Hence $L_{H} G=$ $k L_{G} G k^{-1}=k L_{\text {alg }} G k^{-1}$.

2. Let $L_{\text {qalg }} G$ be a quasi-algebraic subgroup of $L(G, \sigma)$. Then there is some $k \in L(G, \sigma)$ such that $L_{q a l g} G=k L_{\text {alg }} G k^{-1}$. Put $H=k G k^{-1}$ and $\mathfrak{h}=k \mathfrak{g} k^{-1}$ and we have reduced the statement of theorem 4.3 to the lemma 4.6. $\square$

We now give the proof of lemma 4.6:

Proof of Lemma 4.6. Define:

$$
L_{\text {alg }}^{\prime} G:=\left\{e^{t X_{1}} e^{t Y_{1}} \ldots e^{t X_{n}} e^{t Y_{n}} g \mid g \in G, X_{i}, Y_{i} \in \mathfrak{g}, e^{t X_{i}} e^{t Y_{i}}=e\right\} .
$$

We have to show: $L_{a l g}^{\prime} G=L_{\text {alg }} G$.

- We show: $L_{a l g}^{\prime} G \subset L_{a l g} G$. First remark that $L_{\text {alg }}^{\prime} G$ is a group of periodic mappings $c: \mathbb{R} \rightarrow G$ with period 1 . As $g e^{t X}=e^{\operatorname{tAd}(g) X} g$, the product of two elements is again in $L_{\text {alg }}^{\prime} G$. Checking the group axioms is then elementary. Thus $L_{\text {alg }}^{\prime} G$ is a subgroup of $L G$. From theorem 4.7. in [Mit88] it follows that $c(t)=\exp t X \exp t Y$ is in $L_{\text {alg }} G$ iff $\exp t X \exp t Y=e$. As each element in $L_{\text {alg }}^{\prime} G$ is generated by elements in $L_{a l g} G$, we get: $L_{a l g}^{\prime} G \subset L_{a l g} G$.

- We show: $L_{a l g} G \subset L_{a l g}^{\prime} G$. To prove this direction, we study the action of $L_{a l g}^{\prime} G$ on the building. We show:

1. $L_{a l g}^{\prime} G$ acts transitively on the set of chambers.

2. The isotropy group of a chamber is the same for $L_{a l g} G$ and $L_{\text {alg }}^{\prime} G$.

Those two assertions contain the theorem, as for $g \in L_{a l g} G$ we find the existence of a $g^{\prime} \in L_{\text {alg }}^{\prime} G$ such that $g \Delta_{0}=g^{\prime} \Delta_{0}$ for some fixed chamber $\Delta_{0}$. Thus $g^{\prime-1} g \Delta_{0}=\Delta_{0}$. Thus the product $g^{\prime-1} g$ is in the isotropy group of $\Delta_{0}$ with respect to the $L_{\text {alg }} G$-action, called $L_{\text {alg }} G_{\Delta_{0}}$.

Now the second assertion tells us: $g^{\prime-1} g \in L_{\text {alg }}^{\prime} G_{\Delta_{0}}=L_{a l g} G_{\Delta_{0}}$ Set $g^{\prime \prime}:=$ $g^{\prime-1} g \in L_{\text {alg }}^{\prime} G$. Then $g=g^{\prime} g^{\prime \prime} \in L_{\text {alg }}^{\prime} G$. Thus $L_{a l g} G \subset L_{a l g} G^{\prime}$ and the lemma is proved. Thus we are left with checking assertions 1 . and 2.:

- We prove: The isotropy group of a chamber is the same for $L_{a l g} G$ and $L_{\text {alg }}^{\prime} G$. Let $\mathfrak{B}_{\text {alg }}$ be the affine building, associated to $L_{a l g} G, X \in \mathfrak{B}$ a cell of type $I, P_{I}$ its stabilizer in $L G_{\mathbb{C}}$. We know from [Mit88]:

$$
\begin{aligned}
L_{\text {alg }} G \cap P_{I} & =\left\{\bar{h} \in L_{\text {alg }} G \mid h(t) \exp (t X) h^{-1}(1)=\exp (t X)\right\}= \\
& =\left\{\bar{h} \in L_{\text {alg }} G \mid h(t)=\exp (t X) h(1) \exp (-t X)\right\} \subset \\
& \subset L_{\text {alg }}^{\prime} G .
\end{aligned}
$$

The last inclusion is true, as $\exp (t X) h(1) \exp (-t X)=$ $\exp (t X) \exp (t Y) h(1)$ with $Y=-\operatorname{Ad} h(1) X$ and $[h(1), \exp (t X)]=0$. 
- We prove: $L_{\text {alg }}^{\prime} G$ acts transitively on the set of chambers. To this end, we remark that the action of $L_{a l g} G \cap P_{i} \simeq S U(2)$ is transitive on the chambers having the panel corresponding to $i$ in its boundary. Transitivity on the building follows now as every pair of chambers can be connected by a gallery, which we can follow by repeated application of the transitivity on the chambers surrounding a panel.

As those groups are in $L_{\text {alg }}^{\prime} G$, the action of $L_{\text {alg }}^{\prime} G$ is transitive on $\mathfrak{B}-$ the result follows now.

REMARK 4.4. The strategy of this proof is similar in spirit to the amalgam-based local-global constructions in the Kac-Moody theory.

5. Twin cities and Kac-Moody algebras. This section is devoted to the construction of an explicit realization of twin cities via the Adjoint action.

Define the two simplicial complexes:

$$
\begin{aligned}
& \mathfrak{B}^{+}=\left(L\left(G_{\mathbb{C}}, \sigma\right) / B^{+} \times \Delta\right) / \sim, \\
& \mathfrak{B}^{-}=\left(L\left(G_{\mathbb{C}}, \sigma\right) / B^{-} \times \Delta\right) / \sim .
\end{aligned}
$$

In this description $B^{+}$and $B^{-}$denote opposite Borel subgroups, $\Delta$ denotes the fundamental alcove in a fixed torus $\mathfrak{t} \subset \mathfrak{g}$ and $\sim$ is the equivalence relation defined by $\left(f_{1}, Y_{1}\right) \sim\left(f_{2}, Y_{2}\right)$ iff $Y_{1}=Y_{2} \simeq Y$ and $f_{1} \simeq f_{2}(\bmod ($ Fix $\exp t Y))$. Using the Iwasawa decomposition of $L\left(G_{\mathbb{C}}, \sigma\right)$ we get a second description:

$$
\begin{aligned}
& \mathfrak{B}^{+}=\left(L\left(G_{\mathbb{R}}, \sigma\right) / T \times \Delta\right) / \sim, \\
& \mathfrak{B}^{-}=\left(L\left(G_{\mathbb{R}}, \sigma\right) / T \times \Delta\right) / \sim .
\end{aligned}
$$

Furthermore we set

$$
\mathfrak{B}=\mathfrak{B}^{+} \cup \mathfrak{B}^{-}
$$

DeFinition 5.1 (Apartment). By abuse of notation, let $W_{\text {aff }} \subset L\left(G_{\mathbb{C}}, \sigma\right) / B=$ $L\left(G_{\mathbb{R}}, \sigma\right) / T$ be a realization of the affine Weyl group of $G_{\mathbb{C}}, W_{\text {aff }}^{f}:=f W_{\text {aff }} f^{-1}$. An apartment $\mathcal{A}_{f}^{ \pm} \in \mathfrak{B}^{ \pm}$is the simplicial complex

$$
\mathcal{A}_{f}^{ \pm}:=\left(W_{\text {aff }}^{f} \times \Delta\right) / \sim .
$$

Proof.

- To check that the embedding $W_{\text {aff }} \subset G_{\mathbb{C}} / B=G_{\mathbb{R}} / T$ is well defined, let $\mathfrak{t} \subset \mathfrak{g}$ be a maximal Abelian subalgebra. Let $H:=\left\{g \in G \mid g \mathfrak{t} g^{-1}=\mathfrak{t}\right\}$. $H$ is a group. Let $X \in \mathfrak{t}$ be a regular element, $K:=\operatorname{Fix}(X) \simeq T$. Then $W=H / T \subset G / T$. - $\mathcal{A}_{f}^{ \pm}$is a thin Coxeter complex of type $W$. Thus $\mathcal{A}_{f}^{ \pm}$is an apartment.

Lemma 5.1. Two elements $(f, X),(g, Y) \in \mathfrak{B}^{ \pm}$are contained in the same connected component iff $f^{-1} g \in \widehat{L}(G, \sigma)^{ \pm}$.

Proof. This is a restatement of lemma 4.3. 
We want now to embed the twin city in the compact real form $\widehat{L}\left(\mathfrak{g}_{\mathbb{R}}, \sigma\right)$ of a Kac-Moody Lie algebra. It will appear as a tessellation of a space $\mathfrak{H}_{\ell, r}$ defined as the intersection of the sphere of radius $l, l \in \mathbb{R}$, with a horosphere $r_{d}= \pm r$. The two sheets of this sphere will correspond to $\mathfrak{B}^{+}$resp. $\mathfrak{B}^{-}$.

We require that the regularity of $\widehat{L}(G, \sigma)$ is such that the restriction of the gauge action of $L(G, \sigma)$ on $L(\mathfrak{g}, \sigma)$ to $\mathfrak{H}_{\ell, r}$ is polar. This condition is fulfilled for $\widehat{A}_{n} G$ and for $\widehat{M G}^{\sigma}$ as is shown in [Fre09]. For Kac-Moody groups of $H^{1}$-loops acting on the Kac-Moody algebra of $H^{0}$-loops it is a consequence of Terng's work [Ter95].

To construct the embedding, we start with the conjugation action:

$$
\widehat{\varphi}: \widehat{L}(G, \sigma) \times \widehat{L}(G, \sigma) \longrightarrow \widehat{L}(G, \sigma), \quad(g, h) \mapsto g h g^{-1} .
$$

By differentiation we get the adjoint action on the Lie algebra:

$$
\widehat{\varphi}: \widehat{L}(G, \sigma) \times \widehat{L}(\mathfrak{g}, \sigma) \longrightarrow \widehat{L}(\mathfrak{g}, \sigma), \quad(g, \widehat{u}) \mapsto g \widehat{u} g^{-1} .
$$

In contrast to the finite dimensional theory it is not possible to cover $\widehat{L}(\mathfrak{g}, \sigma)$ with maximal conjugate flats.

Nevertheless, the polarity assumption shows this to be possible for the restriction to $\mathfrak{H}_{\ell, r}$ (which is invariant under the adjoint action). Hence we conclude that $\mathfrak{H}_{\ell, r}$ is covered with finite dimensional conjugate Abelian subalgebras. So in the end the situation is exactly as in the finite dimensional case; hence the algebra works out exactly the same way:

We find for a Cartan subalgebra $\widehat{\mathfrak{h}}$

$$
\begin{aligned}
& \widehat{\varphi}: \quad \widehat{L}(G, \sigma) \times \mathfrak{H}_{\ell, r} \quad \longrightarrow \quad \mathfrak{H}_{\ell, r}, \quad(g, \widehat{u}) \mapsto g \widehat{u} g^{-1}, \\
& \widehat{\varphi}: \quad \widehat{L}(G, \sigma) \times \widehat{\mathfrak{h}} \cap \mathfrak{H}_{\ell, r} \quad \longrightarrow \quad \mathfrak{H}_{\ell, r}, \quad(g, \widehat{u}) \mapsto g \widehat{u} g^{-1} .
\end{aligned}
$$

Taking $\widehat{\mathfrak{h}}$ to be the standard Cartan subalgebra (i.e. for non-twisted groups, $\mathfrak{h}$ consists of the two dimensional extension of a Cartan subalgebra of $\mathfrak{G}$, interpreted as constant loops), we find that $\mathfrak{h}_{H}:=\widehat{\mathfrak{h}} \cap \mathfrak{H}_{\ell, r}$ consists of triples $\widehat{X}=\left(X, r_{c}, r_{d}\right)$ where $r_{c}$ is defined by the condition $|\widehat{X}|=l$.

The exponential image of $\widehat{\mathfrak{h}}$ is a torus $\widehat{T} \simeq T \oplus \mathbb{S}^{1} \oplus \mathbb{S}^{1} \subset \widehat{L}(G, \sigma)$. As $\widehat{\mathfrak{h}}$ is fixed by $\widehat{T}$, we get a well defined surjective action

$$
\widehat{\varphi}: \quad \widehat{L}(G, \sigma) / \widehat{T} \times \mathfrak{h}_{H} \quad \longrightarrow \quad \mathfrak{H}, \quad\left(g, u_{H}\right) \mapsto g u_{H} g^{-1} .
$$

The surjectivity of this map follows from the polarity of the adjoint action (see theorem 2.1).

Using the equivalence $\widehat{L}(G, \sigma) / \widehat{T} \simeq L(G, \sigma) / T$ we get:

$$
\varphi: L(G, \sigma) / T \times \mathfrak{h}_{H} \longrightarrow \mathfrak{H}, \quad\left(g, u_{H}\right) \mapsto g u_{H} g^{-1} .
$$

Now the inner automorphisms of $\widehat{\mathfrak{h}}$ are the elements of the affine Weyl group $W_{\text {aff }}:=N(T) / T$, so we may further restrict $\widehat{\mathfrak{h}}_{H}$ to a fundamental domain of the action of $W_{\text {aff }}$, denoted $\Delta$. Then the map

$$
\varphi: L(G, \sigma) / T \times \Delta \quad \longrightarrow \quad \mathfrak{H}, \quad\left(g T, \widehat{u}_{H}\right) \mapsto g \widehat{u}_{H} g^{-1}
$$

is again surjective. 
We can now construct a chamber complex by identifying $\Delta$ with a simplex $\mathfrak{B}$ with boundary and taking its $\widehat{L}(G, \sigma)$-translates.

This construction proves the following theorems:

TheOREM 5.1 (Embedding of the twin city). For each algebra $L(\mathfrak{g}, \sigma)$ there is a 2-parameter family of embeddings for the twin city, parametrized by $r$ and the norm l. Those embeddings are equivariant in the sense that:

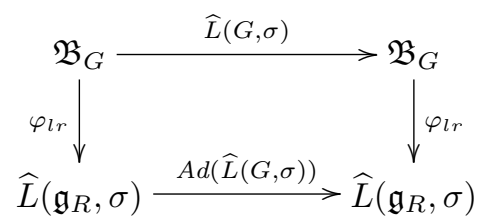

We call this 2-parameter family the thick twin city.

This construction yields the following result:

COROLlary 5.1. Suppose the adjoint action of $\widehat{L}(G, \sigma)$ induces a polar action. Every torus in a Kac-Moody group $\widehat{L}(G, \sigma)$ corresponds to a complete twin apartment of the twin city.

More generally, we have:

THEOREM 5.2. There is a correspondence between twin apartments in the twin city and tori of finite type in $\widehat{L}\left(G_{\mathbb{C}}, \sigma\right)$.

REMARK 5.1. Bertrand Remy proves a similar result for arbitrary algebraic KacMoody groups, showing a correspondence between twin apartments and Cartan subalgebras [Rém02], section 10.4.3. .

Remark 5.2. For Kac-Moody groups of the classical type, theorem 5.2 can be proven by linear representations of the twin cities as complexes of periodic flags in Hilbert spaces [Fre09] for the case $\widetilde{A}_{n}$ and a sketch for the other types and [Fre11a] for the details. The possibility of a similar construction for Kac-Moody groups of the exceptional types is an interesting open problem.

Proof of theorem 5.2. The embedding shows this theorem for twin apartments corresponding to tori in the Kac-Moody group $\widehat{M G}^{\sigma}$. We have to prove two directions:

- Let $A$ be an arbitrary twin apartment in the affine twin building $\Delta_{+} \cup \Delta_{-} \subset$ $\mathfrak{B}^{+} \cup \mathfrak{B}^{-}$. Let $G\left(\Delta_{+}, \Delta_{-}\right)$be the quasi-algebraic group associated to $\Delta_{+} \cup \Delta_{-}$. By Bertrand Remy's result, $A$ corresponds to a torus in $G\left(\Delta_{+}, \Delta_{-}\right)$. The embedding of $G\left(\Delta_{+}, \Delta_{-}\right)$as a subgroup in $\widehat{L}(G, \sigma)$ identifies $A$ with a torus in $\widehat{L}(G, \sigma)$.

- Let $T^{\prime}$ be a torus in $\widehat{L}(G, \sigma)$. As all tori of finite type are conjugate, there is a $g$ such that $T^{\prime}=g T g^{-1}$, where $T$ is the standard torus. $T^{\prime}$ corresponds to the apartment that is the translate by $g$ of the apartment corresponding to $T$.

REMARK 5.3. A second possible proof of theorem 5.2 consists in identifying the stabilizers of twin apartments in $\mathfrak{B}$ and of tori in $\widehat{L}(G, \sigma)$. This is an adaption of the strategy used by Bertram Remy in [Rém02] to our setting. A third proof is implicit in 
the description of apartment systems in section [AB08]. A fourth proof constructs for each apartment a compact real form such that the apartment corresponds to a torus of this compact real form. Then the result follows from corollary 5.1.

Let us now generalize this result to $s$-representations of Kac-Moody symmetric spaces. For an overview of the theory and the definition of Kac-Moody symmetric spaces see [Fre07], for a detailed description see [Fre09]. Their adjoint actions induce s-representations of involutions of affine Kac-Moody algebras. Christian Gross proved in [Gro00] those to be a polar representation for the action of $H^{1}$-Kac-Moody groups on $H^{0}$-Kac-Moody algebras. The result extends to all other regularity conditions, mentioned in this paper especially smooth loops, $k$-differentiable loops and holomorphic loops on $A_{n}$ resp. $\mathbb{C}^{*}$. The last case corresponds to the $s$-representations of Kac-Moody symmetric spaces [Fre09].

Let $\widehat{L}(\mathfrak{g}, \sigma)$ be an affine Kac-Moody algebra of compact type and $\widehat{\rho}$ and involution. Let $\widehat{L}(\mathfrak{g}, \sigma)=\mathcal{K} \oplus \mathcal{P}$ be the decomposition into the \pm 1 -eigenspaces of $\widehat{\rho}$. Let $\widehat{\mathbf{K}}$ be the Kac-Moody group associated to $\mathcal{K}$ and $\mathbf{K}$ its loop group part. The $s$-representation of $\mathbf{K}$ (resp. $\widehat{\mathbf{K}}$ ) is the action of $\mathbf{K}$ on $\mathcal{P}$ induced by the adjoint action of $\widehat{L}(G, \sigma)$ on $\widehat{L}(\mathfrak{g}, \sigma)$ by restriction to $\mathbf{K}$ (resp. $\widehat{\mathbf{K}}$ ) and $\mathcal{P}$. As usual, we allow for $\mathbf{K}, \widehat{\mathbf{K}}, \mathcal{K}$ and $\mathcal{P}$ all regularity conditions, we have introduced so far.

Then the result of Christian Groß[Gro00] and its extension to all other regularity conditions can be stated as follows:

THEOREM 5.3. The s-representation of $\mathbf{K}$ and $\widehat{\mathbf{K}}$ on the Hilbert space $\mathcal{P}_{r, \ell}$ is a polar representation.

Hence $\mathcal{P}_{\ell, r}$ is covered with finite dimensional conjugate Abelian subalgebras $\mathfrak{h}_{i}$. So the situation is exactly the same as in the Kac-Moody group case.

We find for a Cartan subalgebra $\widehat{\mathfrak{h}}$

$$
\widehat{\varphi}: \widehat{L}(G, \sigma) \times \widehat{\mathfrak{h}} \cap \mathcal{P}_{\ell, r} \longrightarrow \mathcal{P}_{\ell, r}, \quad(g, \widehat{u}) \mapsto g \widehat{u} g^{-1}
$$

is surjective. Taking $\widehat{\mathfrak{h}}$ to be the standard Cartan subalgebra (i.e. for non-twisted groups, $\mathfrak{h}$ consists of the two dimensional extension of a Cartan subalgebra of $\mathcal{P}$, interpreted as constant loops), we find that $\mathfrak{h}_{\ell, r}:=\widehat{\mathfrak{h}} \cap \mathcal{P}_{\ell, r}$ consists of triples $\widehat{X}=$ $\left(X, r_{c}, r_{d}\right)$ where $r_{c}$ is defined by the condition $|\widehat{X}|=l$.

Defining $C_{K} \subset \mathbf{K}$ (resp. $\widehat{C}_{K} \subset \widehat{\mathbf{K}}$ ) to be the centralizer of $\widehat{\mathfrak{h}}$ we get a well defined surjective action

$$
\widehat{\varphi}: \quad \widehat{L}(G, \sigma) / \widehat{C}_{K} \times \mathfrak{h}_{\ell, r} \quad \longrightarrow \mathcal{P}_{\ell, r}, \quad\left(g, u_{H}\right) \mapsto g u_{H} g^{-1} .
$$

The surjectivity of this map follows from the polarity of the adjoint action (see theorem 2.1).

Using the equivalence $\widehat{\mathbf{K}} / \widehat{C}_{K} \simeq \mathbf{K} / C_{K}$ we get:

$$
\varphi: \mathbf{K} / C_{K} \times \mathfrak{h}_{\ell, r} \longrightarrow \mathcal{P}_{\ell, r}, \quad\left(g, u_{H}\right) \mapsto g u_{H} g^{-1} .
$$

Now the inner automorphisms of $\widehat{\mathfrak{h}}$ are the elements of the affine Weyl group $W_{\text {aff }}:=N_{K}(\mathfrak{h}) / C_{K}(\mathfrak{h})$, where $N_{K}(\mathfrak{h})$ denotes the normalizer of $\mathfrak{h}$, so we may further restrict $\widehat{\mathfrak{h}}_{\ell, r}$ to a fundamental domain $\Delta_{W}$ for the action of $W_{\text {aff. Then the map }}$

$$
\varphi: \mathbf{K} / C_{K} \times \Delta \longrightarrow \mathcal{P}_{\ell, r}, \quad\left(g T, \widehat{u}_{H}\right) \mapsto g \widehat{u}_{H} g^{-1}
$$


is again surjective.

Using the description $\mathfrak{B}=\left(\mathbf{K} / C_{K} \times \Delta\right) / \sim$ and identifying $\Delta_{W}$ with a fundamental simplex $\Delta$ of the city, we get the embedding result:

THEOREM 5.4 (Embedding of the twin city). For each subspace $\mathcal{P} \subset L(\mathfrak{g}, \sigma)$ there is a 2-parameter family of embeddings for the twin city, parametrized by $r$ and the norm $\ell$. Those embeddings are equivariant in the sense that:

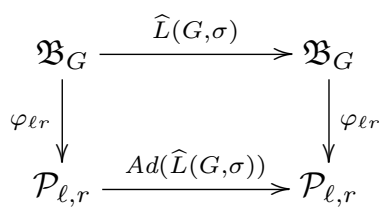

We got explicit realization for the affine twin cities in tangential spaces of KacMoody symmetric spaces, thus showing, that twin cities appear "in nature".

6. Topology and geometry of $\mathfrak{B}$. There are three sundry ways to define a topology (resp. geometry) on the twin cities:

1. A structure on the geometric realization of $\mathfrak{B}$.

2. A structure on the set of chambers in $\mathfrak{B}$.

3. A structure on the set of buildings in $\mathfrak{B}$.

We will discuss these 3 ways in the following three subsections.

6.1. The structure on the geometric realization of $\mathfrak{B}$. The embedding of the twin city into spaces $H_{\ell, r}$ shows:

Theorem 6.1. Let $\widehat{L}(G, \sigma)$ be a Hilbert-, Banach- or Fréchet-Lie group. The geometric realization of the positive (resp. negative) component $\mathfrak{B}^{+}$(resp. $\mathfrak{B}^{-}$) of the twin city carries the same structure.

Using the results about the analytic structure of the various Kac-Moody groups, as developed in [PS86], [Ter95], [Pop05], and [Fre09], we get the following corollary:

COROllary 6.1 (The most important examples).

1. Each city associated to $\widehat{M G}$ carries a natural tame Fréchet structure.

2. Each city associated to $\widehat{A_{n} G}$ carries a natural Banach space structure.

3. Each city associated to $\widehat{L^{\infty} G}$ carries a natural tame Fréchet structure.

4. Each city associated to $\widehat{L^{1} G}$ carries a natural Hilbert space structure.

Using the description of $\widehat{M g}$ as inverse limit of the algebras $\widehat{A_{n} \mathfrak{g}}$ [Omo97] and [Fre09], we find this structure reflected in an inverse limit system $\left\{\mathfrak{B}_{\widehat{M G}}, \lim _{\longleftarrow} \mathfrak{B}_{A_{n} G}\right\}$.

Thus the twin city for $\widehat{M G}$ is surrounded by a cloud of buildings corresponding to groups of weaker regularity.

6.2. The structure on the set of chambers in $\mathfrak{B}$. As chambers in $\mathfrak{B}$ correspond bijectively to elements in the quotient $M G / T$, the space of chambers inherits the tame Fréchet topology of $M G / T$. Study the gauge action of $M G$ on $M \mathfrak{g}$. By theorem 2.1 it is a polar action. Let $X \in \mathfrak{t}$ be an element in the Lie algebra of $T$, such that $\{M G \cdot X\}$ is a principal orbit. As the stabilizer of $X$ is $T$, we have $\{M G \cdot X\} \simeq M G / T$.

Hence the space of chambers can be identified with an isoparametric submanifold. So the structure of the space of chambers is well understood. 
THEOREM 6.2. Let $S$ be an isoparametric PF-submanifold of a Hilbert space. Suppose $S$ is homogeneous and it is the principal orbit of the gauge action of a Hilbert loop group $L^{1} G^{\sigma}$. Then the points in the isoparametric submanifold correspond bijectively to chambers in the associated city. Furthermore curvature spheres correspond to panels.

For the definition of isoparametric submanifolds see [Ter89] and [PT88]. All known isoparametric submanifolds with higher codimension are of this type.

Conversely Ernst Heintze and Xiaobo Liu [HL99] — prove the following theorem:

TheOrem 6.3. A complete, connected, full, irreducible isoparametric submanifold $M$ of an infinite dimensional Hilbert space $V$ with codimension $\neq 1$ is a principal orbit of a polar action.

The set $Q$ constructed in [HL99] has the structure of an affine algebraic building. For more details [Ter95], [HPTT95], [Fre11b], and the references therein. It is conjectured that all polar actions on Hilbert spaces correspond to $P(G, H)$ actions under suitable assumptions on the cohomogeneity. Very promising partial results in this direction due to Claudio Gorodski, Ernst Heintze and Kerstin Weinl exist. If this is the case one gets an equivalence between cities and isoparametric submanifolds of codimension $\neq 1$ mirroring the situation described by Thorbergsson's theorem in the finite dimensional situation - [Tho91].

6.3. The structure on the set of buildings in $\mathfrak{B}$. While the space of chambers and the simplicial realization allow a metric structure similar to the one of the subjacent Lie group, i.e. a Hilbert, Banach or Fréchet space structure, the situation is completely different for $\mathfrak{B}$ itself. The simple fact that the chambers belonging to a single building are dense in the space of all chambers shows that no refinement of a topology on the space of chambers will give a topology on the space of buildings.

As we choose to define the twin city in terms of the geometric Kac-Moody groups, we will also describe the geometry and topology of those groups. We want two buildings in $\mathfrak{B}$ to be close iff there is a small group $\widehat{L}(G, \sigma)$, i.e. a group defined using strong regularity conditions, containing both of them. As the product of two functions of a given regularity is of the same regularity, we find that a distance defined in this way will be ultrametric.

Hence we will show that a twin city carries a ultrametric pseudo distance.

For $\widehat{f} \in \widehat{L}(G, \sigma)$ let $f=\sum a_{k} z^{k}$ be the (matrix valued) associated Fourier series of the loop part. Recall that convergence conditions on the series $\sum\left|a_{k}\right|$ correspond to regularity conditions on $f$ [GW84] for an extensive overview:

- $f$ is in $L^{r} G$ iff $\sum\left|a_{k}\right| k^{r}<\infty$.

- $f$ is smooth iff $\sum\left|a_{k}\right| k^{r}<\infty \quad \forall r \in \mathbb{N}$.

- $f$ is holomorphic on $A_{n}$ iff $\sum\left|a_{k}\right| e^{k n}<\infty$.

- $f$ is holomorphic on $\mathbb{C}^{*}$ iff $\sum\left|a_{k}\right| e^{k n}<\infty \quad \forall n \in \mathbb{N}$.

At the moment of this writing, it is unclear if there is a suitable distance function which is meaningful in the whole range of regularity conditions.

$$
\text { The }\left\{\boldsymbol{A}_{n}, \mathbb{C}^{*}\right\} \text {-setting. }
$$

To metrize the "cloud" of buildings surrounding the tame twin city associated to a Kac-Moody symmetric space, we propose the following definition: 
Definition 6.1. For $\Delta_{0}, \Delta_{1} \in \mathfrak{B}^{ \pm}$and $x \in \Delta_{0}, y \in \Delta_{1}$ define

$$
\nu(x, y)=\max _{n}\left\{\text { There is } f \in A_{n} G \text { such that } f(x)=y\right\}
$$

and $d(x, y)=e^{-\nu(x, y)}$. Then we put $d\left(\Delta_{0}, \Delta_{1}\right)=d(x, y)$.

This is equivalent to

$\nu(x, y)=\max _{n}\left\{\right.$ There is a function $f$ such that $f(x)=y$ satisfying $\left.\sum\left|a_{k}\right| e^{k n}<\infty\right\}$.

Lemma 6.1 (pseudo distance). $d$ is a ultrametric pseudo distance on the space of buildings in $\mathfrak{B}^{\epsilon}$.

Proof of Lemma 6.1.

1. We prove that $d$ is a ultrametric pseudo distance on the space of chambers. To this end let $x, y, z \in \mathfrak{B}^{\epsilon}$ be chambers. We have to check:

- symmetry: $f \in A^{n} G \Leftrightarrow f^{-1} \in A^{n} G$. Thus $\nu(x, y)=\nu(y, x)$ and $d(x, y)=d(y, x)$.

- strong $\Delta$-inequality: Let $d(x, y)=e^{-\nu(x, y)}, d(y, z)=e^{-\nu(y, z)}$. Thus there is a function $f_{x y} \in A^{\nu(x, y)} G$ such that $f(x)=y$ and a function $f_{y z} \in A^{\nu(y, z)} G$ such that $f(y)=z$. Without loss of generality suppose $\nu(x, y) \leq \nu(y, z)$. Thus $A^{\nu(x, y)} G \supset A^{\nu(y, z)} G$. So $f_{x z}=f_{x y} f_{y z} \in$ $A^{\nu(x, y)} G$. Thus $d(x, z)=e^{-\nu(x, z)} \leq e^{-\nu(x, y)}=d(x, y)$.

2. We have to check that the distance on the space of buildings is well defined. To this end let $x, x^{\prime} \in \Delta_{0}$. There is a quasi-algebraic subgroup $G\left(\Delta_{0}\right)$ acting transitively on $\Delta_{0}$. Let $h \in G\left(\Delta_{0}\right)$ such that $x^{\prime}=h(x)$. Clearly $d\left(x, x^{\prime}\right)=0$. The result follows now from the triangle inequality.

The Hilbert space setting: $\boldsymbol{H}^{1}$-loops acting on $\boldsymbol{H}^{0}$-spaces. In many papers describing the geometry of Kac-Moody groups (see [HPTT95], [Ter89], and [Ter95]), the setting of $H^{1}$-loops with values in a compact simple Lie group $G$, acting on the space of $H^{0}$-loops in $\mathfrak{g}$, is used. Our results carry over to this setting:

Nevertheless, describing $\mathfrak{B}^{ \pm}=(L G \times \Delta) / \sim$, it seems meaningful to make some changes in the definition of the pseudo distance. As we defined it, the distance between two buildings depends on the convergence radius of the functions transforming one building into the other. For $H^{1}$-functions this definition is useless: The space of buildings such that the distance is 0 is just to big. So it seems meaningful to introduce another distance function:

Definition 6.2 ( $H^{1}$-distance). Let $\Delta_{1}, \Delta_{2} \in \mathfrak{B}^{\epsilon}$. Let $f B^{\epsilon} \in \Delta_{1}, g B^{\epsilon} \in \Delta_{2}$ and let $f g^{-1}=\sum a_{k} e^{i k t}$ be the Fourier series expansion. Then $\nu_{r}\left(f B^{\epsilon}, g B^{\epsilon}\right)=$ $\max _{r}\left\{\sum k^{r} a_{k}<\infty\right\}$ and $d_{r}\left(\Delta_{1}, \Delta_{2}\right)=e^{-\nu\left(f B^{\epsilon}, g B^{\epsilon}\right)}$.

Lemma 6.2. The $H^{1}$-distance is a ultrametric pseudo distance.

Proof. The proof follows the pattern of the proof for lemma 6.1.

7. An example: Type $\boldsymbol{A}_{1}^{(1)}$. The affine Kac-Moody algebra $\widehat{L} \mathfrak{s l}(2, \mathbb{C})$ is the two dimensional extension by a derivation and a central element of the algebra of $2 \pi$-periodic $H^{0}$-Sobolev functions on $\mathbb{R}$ into the simple Lie algebra $\mathfrak{s l}(2, \mathbb{C}) . \mathfrak{s l}(2, \mathbb{C})$ is a 3-dimensional complex Lie algebra of rank 1. Choose generators $e, f$ and $h$ of 
$\mathfrak{s l}(2, \mathbb{C})$ satisfying the usual commutation relations

$$
\begin{aligned}
& {[h, e]=e} \\
& {[h, f]=-f} \\
& {[e, f]=h .}
\end{aligned}
$$

Each element $g=\left(g_{h}, g_{e}, g_{f}\right) \in L \mathfrak{s l}(2, \mathbb{C})$ has a Fourier series expansion $g=\sum_{n} g_{n} t^{n}$ such that $g_{n}=\left(g_{h, n}, g_{e, n}, g_{f, n}\right)$. The subalgebra of algebraic loops

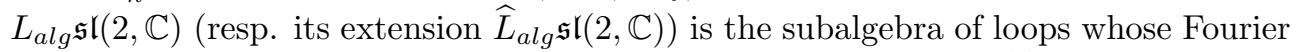

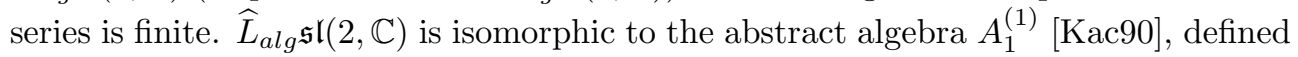
as the realization $\mathfrak{g}(A)$ of the affine Cartan matrix

$$
A=\left(\begin{array}{rr}
2 & -2 \\
-2 & 2
\end{array}\right)
$$

A basis for $L \mathfrak{s l}(2, \mathbb{C})$ is given by $\left(e \cdot t^{n}, h \cdot t^{n}, f \cdot t^{n}, n \in \mathbb{Z}\right)$. $\mathfrak{s l}(2, \mathbb{C})$ can be embedded into $\widehat{L} \mathfrak{s l}(2, \mathbb{C})$ as the subalgebra of constant loops. For a constant function $g(t) \equiv g_{0}$ the derivative $g^{\prime}(t)=0$ vanishes. In consequence the 2-form $\omega$ (compare 2), describing the $c$-component of the Lie bracket of two functions, vanishes on elements of $\mathfrak{s l}(2, \mathbb{C}) \subset \widehat{L} \mathfrak{s l}(2, \mathbb{C})$. Hence $\mathfrak{s l}(2, \mathbb{C})$ is a subalgebra.

$\mathfrak{h}=\mathbb{C} h$ is a Cartan subalgebra in $\mathfrak{s l}(2, \mathbb{C})$. The relation $[d, g]=0$ for $g$ any constant function together with $c$ being central makes $\widehat{h}=\mathfrak{h} \oplus \mathbb{C} c \oplus \mathbb{C} d$ a Cartan subalgebra in $L \mathfrak{s l}(2, \mathbb{C})$. All further Cartan subalgebras of $L \mathfrak{s l}(2, \mathbb{C})$ are conjugate to $\widehat{\mathfrak{h}}$.

Let $\widehat{L} S L(2, \mathbb{C})$ be the corresponding affine complex Kac-Moody group. This group has a structure as a torus bundles over the loop group $L S L(2, \mathbb{C})$. Details of the technical construction may be found in [PS86]. Let $\widehat{B}$ be the standard positive Borel subgroup of $\widehat{L} S L(2, \mathbb{C})$ that is, $\widehat{B}$ is the subgroup of $\widehat{L} S L(2, K)$ corresponding to loops whose Fourier coefficients are non-negative and whose constant term is upper triangular. Hence a loop $f \in \widehat{L} S L(2, \mathbb{C})$ is in $\widehat{B}$ iff $f=\sum_{n \geq 0} f_{n} t^{n}$ such that

$$
f_{n}=\left(\begin{array}{ll}
f_{n, 11} & f_{n, 12} \\
f_{n, 21} & f_{n, 22}
\end{array}\right) \text { and } f_{0}=\left(\begin{array}{cc}
f_{n, 11} & f_{n, 12} \\
0 & f_{n, 22}
\end{array}\right) \text { is upper triangular. }
$$

Similarly we denote by $\widehat{L}_{\text {alg }} S L(2, \mathbb{C})$ the subgroup of algebraic loops. By construction, the loop group $L_{a l g} S L(2, \mathbb{C})$ of this group is isomorphic to the linear algebraic group corresponding to the affine algebraic group scheme $S L_{2}$ evaluated over the ring $\mathbb{C}\left[t, t^{-1}\right]$. The affine Weyl group of the group $\widehat{L} S L_{\text {alg }}(2, \mathbb{C})$ is the reflection group

$$
W \equiv W_{\text {aff }}=\left\langle s, t \mid s^{2}=t^{2}=1\right\rangle \cong \mathbb{Z} \rtimes\{ \pm 1\} .
$$

The adjoint action of $\widehat{L} S L_{\text {alg }}(2, \mathbb{C})$ on $\widehat{L} \mathfrak{s l}(2, \mathbb{C})$ preserves the subalgebra $\widehat{L}_{\text {alg }} \mathfrak{s l}(2, \mathbb{C})$.

Let us now turn to the compact real forms: The compact real form of $\widehat{L} \mathfrak{s} l(2, \mathbb{C})$ is $\widehat{L} \mathfrak{s u}(2)$, the two dimensional extension of the algebra of $2 \pi$-periodic loops into $\mathfrak{s u}(2)$. Similarly, $\widehat{L} S U(2)$ is the - up to conjugation unique - compact real form of $\widehat{L} S L(2, \mathbb{C})$. We define the subalgebra (resp. subgroup) of algebraic loops as in the complex case. The Adjoint action of $\widehat{L} S U_{\text {alg }}(2)$ on $\widehat{L} \mathfrak{s u}(2)$ fixes the subalgebra $\widehat{L}_{\text {alg } \mathfrak{s u}}(2)$. 
There is a unique $A d$-invariant scalar product on $\widehat{L} \mathfrak{s u}(2)$, defined as follows:

$$
\begin{aligned}
& \langle g, h\rangle=\int_{0}^{2 \pi}\langle g(t), h(t)\rangle d t \quad \text { for } f(t), g(t) \in L \mathfrak{s u}(2) \\
& \langle c, d\rangle=-1 \\
& \langle d, d\rangle=\langle c, c\rangle=0 .
\end{aligned}
$$

This is a Lorentz scalar product. $c$ and $d$ define lightlike directions, $L \mathfrak{s u}(2)$ is spacelike while $c+d$ is a timelike direction. Cartan subalgebras of this Lie algebra are 3-R्R-dimensional. Let $\widehat{\mathfrak{h}}=\mathfrak{h} \oplus \mathbb{R} c \oplus \mathbb{R} d$ denote the standard Cartan subalgebra in $L \mathfrak{s u}(2)$. The restriction of $\langle\quad, \quad\rangle$ is a Lorentz scalar product. The space $\mathfrak{h}_{\ell, r}:=\{x \in$ $\left.\widehat{\mathfrak{h}}|| x \mid=l, r_{d}= \pm r\right\}$ consists of the two components, $\mathfrak{h}_{\ell, r}^{+}=\left\{x \in \mathfrak{h}_{\ell, r}, r_{d}>0\right\}$ and $\mathfrak{h}_{\ell, r}^{-}=\left\{x \in \mathfrak{h}_{\ell, r}, r_{d}<0\right\}$, each isomorphic to $\mathbb{R}$ and each one preserved by the adjoint action. Let $r_{d}= \pm 1$ and define an isometry $\varphi: \mathbb{R} \longrightarrow \mathfrak{h}_{\ell, 1}^{+}, 0 \mapsto(0,-l, 1)$. The Weyl group is generated by the reflections $s_{0}$ at $\varphi(0)$ and $s_{1}$ at $\varphi(1)$. Hence

$$
\begin{aligned}
& s_{0}(\varphi(x))=\varphi(-x), \\
& s_{1}(\varphi(x))=\varphi(2-x) .
\end{aligned}
$$

A fundamental domain for the Weyl group action is thus the interval $[\varphi(0), \varphi(1)] \subset$ $\mathfrak{h}_{\ell, r}$.

In the sequel we will work out the embedding construction for non algebraic loop groups. Performing the construction for nonalgebraic groups yields twin cities, performing them for the algebraic groups yields a single twin building.

The building associated to $L S L(2, \mathbb{C})$ is an $\mathbb{R}$-tree. It can be described as

$$
\mathfrak{B}=(L S L(2) / B \times \Delta) / \sim,
$$

where $\Delta$ denotes a fundamental simplex. In the case of $L S L(2, \mathbb{C})$ the fundamental simplex $\Delta$ consists of a 1 -simplex $(0,1)$ and two vertices $(0)$ and (1) in its boundary. The equivalence relation $\sim$ describes the stabilizers of the various cells in the fundamental simplex. Hence with $s_{0}^{\prime}$ denoting the reflection at the vertex (0) and $s_{1}^{\prime}$ denoting the reflection at the vertex (1) in the standard apartment, we get:

1. On the 1-simplex, the equivalence relation is trivial, i.e. $(f B,(0,1)) \sim$ $(g B,(0,1))$ iff $f g^{-1} \in B$.

2. On the simplex (0) two elements $(f B,(0)) \sim(g B,(0))$ iff $f g^{-1} \in P_{s_{0}^{\prime}}=$ $B \cup B s_{0}^{\prime} B$.

3. On the simplex (1) two elements $(f B,(1)) \sim(g B,(1))$ iff $f g^{-1} \in P_{s_{1}^{\prime}}=$ $B \cup B s_{1}^{\prime} B$.

The Iwasawa decomposition $L S L(2, \mathbb{C})=L S U(2) A N$ where $A$ is diagonal with constant coefficients and $N$ consists of all functions $g \in L S L(2, \mathbb{C})$ whose Fourier series $g=\sum_{n \geq 0} g_{n} t^{n}$ has only positive coefficients and additionally

$$
g_{0}=\left(\begin{array}{cc}
1 & g_{0,12} \\
0 & 1
\end{array}\right)
$$

Using furthermore $B=T A N$, where $\mathrm{T}$ is a torus in the compact real form, this yields the equivalence

$$
L S L(2, \mathbb{C}) / B=L S U(2) / T .
$$


Hence we get for the building the equivalent description:

$$
\mathfrak{B}=(L S U(2) / T \times \Delta) / \sim .
$$

Using the decomposition of parabolic subgroups $P_{s_{i}}$ in products of their respective Levi components $L_{s_{i}}$ with Borel subgroups [Gar97], we can describe the equivalence relation $\sim$ again explicitly.

1. On the 1-simplex, the equivalence relation is trivial, i.e. $(f T,(0,1)) \sim$ $(g T,(0,1))$ iff $f g \in T$.

2. On the simplex $(0)$ two elements $(f T,(0)) \sim(g T,(0))$ iff $f g^{-1} \in L_{s_{0}^{\prime}}=$ $T \cup T s_{0}^{\prime} T$.

3. On the simplex (1) two elements $(f T,(1)) \sim(g T,(1))$ iff $f g^{-1} \in L_{s_{1}^{\prime}}=$ $T \cup T s_{1}^{\prime} T$.

Define now the 2-parameter family $\varphi_{\ell, r}$ of embeddings of the Tits building in the Kac-Moody algebra as follows:

1. The vertex $(I d,(0))$ is mapped to the coordinate $v_{0}=\left((0,0,0), \frac{-\ell}{r}, r\right)$. Here the first coordinate denotes the point in the loop algebra, the second the $c$-coefficient $r_{c}$ and the third the $d$-coefficient $r_{d}$. The vertex $\left(g P_{s_{0}},(0)\right)$ is mapped onto $\operatorname{Ad}(g)\left(v_{0}\right)$. It is straight forward, to check that this is well defined. As the $d$-coefficient is preserved by the adjoint action and the $c$ coefficient may be reconstructed by the isometry condition, it is sufficient to calculate the loop coefficient. We have the formula [Ter95]

$$
A d\left(g L_{s_{0}}\right)\left(v_{0}\right)=g v_{0} g^{-1}-r g^{\prime} g^{-1}=r g^{\prime} g^{-1} .
$$

2. We do a similar job for $\left(g P_{s_{1}},(1)\right)$, mapping $(I d,(1))$ onto $v_{0}=$ $\left((1,0,0),-\frac{\ell-1}{r}, r\right)$, where $(1,0,0)$ describes the element in the basis $(h, e, f)$ of $\mathfrak{s u}(2)$.

$$
A d\left(g L_{s_{1}}\right)\left(v_{0}\right)=g v_{0} g^{-1}-r g^{\prime} g^{-1}=r g^{\prime} g^{-1} .
$$

3. The cell $(I d,(0,1))$ is mapped onto the interval $e_{0}=\left((\alpha, 0,0), 1-\frac{\|\alpha\|}{r}, r\right)$. The cell $\left(g L_{s_{0}}\right)$ is mapped onto the translates

$$
A d(g T)\left(e_{0}\right)=g e_{0} g^{-1}-r g^{\prime} g^{-1} .
$$

Let $X$ denote the Tits building. Then for each $r>0, \varphi_{0, r}(X)$ lies in the future lightcone of the Kac-Moody algebra $\mathfrak{G}$. In particular, $\varphi_{0, r}(X)$ lies in the intersection of the lightcone of $\mathfrak{G}$ with the the plane $r_{d}=r$, while $\varphi_{0,-r}(X)$ lies in the past lightcone. Moreover, $\varphi_{-1, r}(X)$ is future directed timelike and $\varphi_{-1,-r}(X)$ is past directed timelike, while $\varphi_{1, \pm r}(X)$ are spacelike. The images of the 2-parameter family fill thus the whole Kac-Moody algebra with the exception of the subspace $\left\{r_{d} \equiv 0\right\}$, where $r_{d}$ denotes as usual the coefficient of $d$. This subspace contains images of the spherical building at infinity of $\mathfrak{B}$. We will study this topic in a sequel to this article.

\section{REFERENCES}

[AB08] P. Abramenko And K. Brown, Buildings, volume 248 of "Graduate Texts in Mathematics", Springer Verlag, New York, 2008.

[AR98] P. AbRAMENKo AND M. RonAN, A characterization of twin buildings by twin apartments, Geometriae Dedicata, 73 (1998), pp. 1-9. 
[BCO03] J. Berndt, S. Console, And C. Olmos, Submanifolds and holonomy, volume 434 of "Research Notes in Mathematics", Chapman \& Hall, Boca Raton, 2003.

[BH99] M. R. BRIDSON AND A. HAEFLIGER, Metric spaces of non-positive curvature, volume 98 of "Grundlehren der mathematischen Wissenschaften", Springer Verlag, Berlin, 1999.

[Cap09] P.-E. Caprace, "Abstract" homomorphisms of split Kac-Moody groups, Mem. amer. Math. Soc., 198 (924):xvi+84, 2009.

[Car02] R. CARTER, Lie Algebras of Finite and Affine Type, volume 96 of "Cambridge studies in advances mathematics", Cambrigde university press, Cambridge, 2002.

[Fre07] W. FREYN, A general theory of affine Kac-Moody symmetric spaces, Kongressberichte der Süddeutschen Geometrietagung, 32 (2007), pp. 4-18.

[Fre09] W. Freyn, Kac-Moody symmetric spaces and universal twin buildings, PhD thesis, Universität Augsburg, 2009.

[Fre11a] W. Freyn, Functional analytic methods for cities, submitted, 2011.

[Fre11b] W. FreYn, Kac-Moody geometry, accepted, 2011.

[Gar97] P. Garrett, Buildings and Classical Groups, Chapman Hall, London, 1997.

[Gro00] C. Gross, s-Representations for involutions on affine Kac-Moody algebras are polar, Manuscripta math., 103 (2000), pp. 339-350.

[Gue97] M. Guest, Harmonic Maps, Loop Groups and Integrable Systems, volume 38 of "London Mathematical Society Student Texts", London mathematical society, London, 1997.

[GW84] R. GoOdman AND N. WALlach, Structure and unitary cocycle representations of loop groups and the group of diffeomorphisms of the circle, Jour. Reine Angew. Mathematik, 347 (1984), pp. 69-133.

[Hei06] E. Heintze, Towards symmetric spaces of affine Kac-Moody type, Int. J. Geom. Methods Mod. Phys., 3:5-6 (2006), pp. 881-898.

[HL99] E. HeIntze AND X. LiU, Homogeneity of infinite-dimensional isoparametric submanifolds, Ann. of Math. (2), 149:1 (1999), pp. 149-181.

[HPTT95] E. Heintze, R. S. Palais, C.-L. Terng, and G. Thorbergsson, Hyperpolar actions on symmetric spaces, pp. 214-245, Conf. Proc. Lecture Notes Geom. Topology, IV. Int. Press, Cambridge, MA, 1995.

[Kac90] V. G. KAC, Infinite-dimensional Lie algebras, Cambridge University Press, Cambridge, third edition, 1990.

[Kob11] S. KobAYASHI, Real forms of complex surfaces of constant mean curvature, Trans. Amer. Math. Soc., 363:4 (2011), pp. 1765-1788.

[Kum02] S. KumAR, Kac-Moody groups, their flag varieties and representation theory, volume 204 of "Progress in Mathematics", Birkhäuser Boston Inc., Boston, MA, 2002.

[KW09] B. Khesin And R. Wendt, The Geometry of Infinite-dimensional Groups, Springer Verlag, Berlin, 2009.

[Mit88] S. A. MitcheLL, Quillen's theorem on buildings and the loops on a symmetric space, L'Enseignement Mathematique, 34 (1988), pp. 123-166.

[MP95] R. V. Moody ANd A. PiAnzola, Lie algebras with triangular decomposition, John Wiley Sons, New York, 1995.

[Omo97] H. Omori, Infinite dimensional Lie groups, American Matheamtical Society, Providence, 1997. Translations of Mathematical Monographs, Vol. 158.

[Pop05] B. Popescu, Infinite dimensional symmetric spaces. Thesis. University of Augsburg, Augsburg, 2005.

[Pop06] B. POPESCU, On the geometry of infinite dimensional Lie groups, preprint, 2006.

[PS86] A. Pressley and G. Segal, Loop groups, Oxford Mathematical Monographs. The Clarendon Press Oxford University Press, New York, 1986. Oxford Science Publications.

[PT88] R. Palais And C.-L. Terng, Critical Point Theory and Submanifold Geometry, volume 1353 of "Lecture Notes in Mathematics", Springer Verlag, Berlin, 1988.

[Rém02] B. RÉmy, Groupes de Kac-Moody déployés et presque déployés, Société mathematiques de France, Paris, 2002.

[Ron03] M. Ronan, Affine Twin Buildings, J. London Math. Soc (2), 68 (2003), pp. 461-476.

[RT94] M. RonAN AND J. Tits, Twin trees I, Inv. math., 116 (1994), pp. 463-479.

[RT99] M. RonAn AND J. TITs, Twin trees II: Local structure and a universal construction, Is. Jour. of math., 109 (1999), pp. 349-377.

[SW85] G. Segal and G. Wilson, Loop groups and equations of KdV type, Publications Mathématiques de l'IHÉS, 61:1 (1985), pp. 5-65.

[Ter89] C.-L. Terng, Proper Fredholm submanifolds of Hilbert space, J. Differential Geom., 
29:1 (1989), pp. 9-47.

[Ter95] C.-L. Terng, Polar actions on Hilbert space, J. of Geom., 5:1 (1995), pp. 129-150.

[Tho91] G. Thorbergsson, Isoparametric foliations and their buildings, Ann. of Math., 133 (1991), pp. 429-446.

[Tit84] J. TITs, Groups and group functors attached to Kac-Moody data, volume 1111 of "Lecture notes in mathematics", pp. 193-233. Springer Verlag, 1984.

[Tsv03] A. Tsvelik, Quantum Field Theory in Condended Matter Physics, Cambridge University Press, Cambridge, 2003.

[Wat79] W. C. WATERHouse, Introduction to Affine Group Schemes, volume 66 of "Graduate texts in Mathematics", Springer Verlag, New York, 1979. 


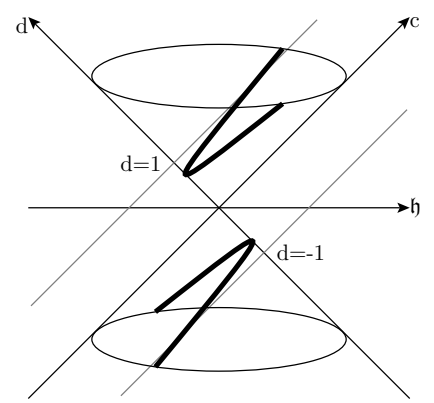

FIG. 1. This figure shows $H_{0,1}$. The fat line is the intersection of the lightcone with the planes $r_{d}= \pm 1$

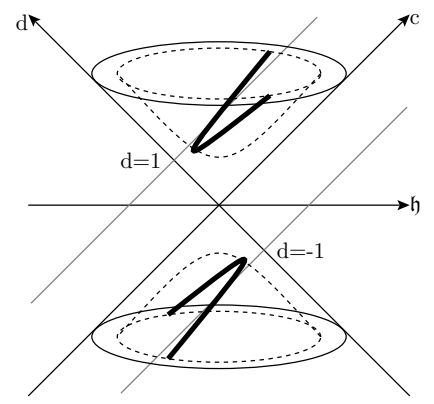

FIG. 2. This figure shows $H_{-1,1}$. The dotted lines represent the sphere of radius -1 . The fat line is the intersection with the planes $r_{d}= \pm 1$

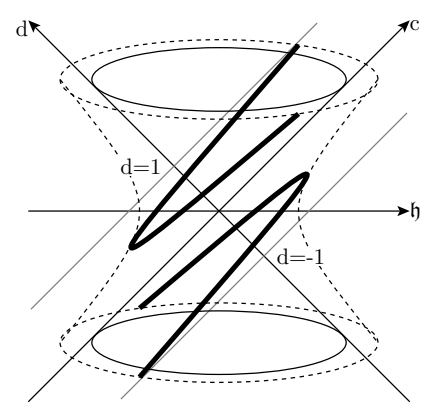

FIG. 3. This figure shows $H_{1,1}$. The dotted lines represent the sphere of radius 1 . The fat line is the intersection with the planes $r_{d}= \pm 1$ 